\title{
GRAPH PARTITIONING USING MATRIX VALUES FOR PRECONDITIONING SYMMETRIC POSITIVE DEFINITE SYSTEMS *
}

\author{
EUGENE VECHARYNSKI ${ }^{\dagger}$, YOUSEF SAAD ${ }^{\ddagger}$, AND MASHA SOSONKINA ${ }^{\S}$
}

\begin{abstract}
Prior to the parallel solution of a large linear system, it is required to perform a partitioning of its equations/unknowns. Standard partitioning algorithms are designed using the considerations of the efficiency of the parallel matrix-vector multiplication, and typically disregard the information on the coefficients of the matrix. This information, however, may have a significant impact on the quality of the preconditioning procedure used within the chosen iterative scheme. In the present paper, we suggest a spectral partitioning algorithm, which takes into account the information on the matrix coefficients and constructs partitions with respect to the objective of enhancing the quality of the nonoverlapping additive Schwarz (block Jacobi) preconditioning for symmetric positive definite linear systems. For a set of test problems with large variations in magnitudes of matrix coefficients, our numerical experiments demonstrate a noticeable improvement in the convergence of the resulting solution scheme when using the new partitioning approach.
\end{abstract}

Key words. Graph partitioning, iterative linear system solution, preconditioning, CauchyBunyakowski-Schwarz (CBS) constant, symmetric positive definite, spectral partitioning

AMS subject classifications. 15A06, 65F08, 65F10, 65N22

1. Introduction. Partitioning of a linear system for its parallel solution typically aims at satisfying two standard objectives: minimizing communication volume and maintaining load balance among different processors. Both of these requirements are motivated by efficiency considerations of the parallel matrix-vector product, which lie in the heart of the iterative solution methods. Once performed the partitioning is then exploited to construct a parallel preconditioner - another crucial ingredient which contributes to the overall performance of the solver. However, the quality of the resulting preconditioner may depend significantly on the given partitioning, which generally targets the efficiency of the parallel matrix-vector multiplication, but ignores its effect on the resulting preconditioner. This preconditioner can be of poor quality especially in the cases when the coefficient matrices have entries with large variations in magnitudes.

For the purpose of obtaining an effective preconditioner, we suggest to remove the requirement on the communication volume and, instead, consider partitionings that favor the quality of the preconditioner. In particular, we focus on the nonoverlapping additive Schwarz (AS) preconditioners for symmetric positive definite (SPD) linear systems $[36,39]$. The proposed partitioning algorithm aims at optimizing the quality of the AS procedure by attempting to minimize the condition number of the

${ }^{*}$ This work was supported in part by Iowa State University under the contract DE-AC0207CH11358 with the U.S. Department of Energy and by the Director, Office of Science, Division of Mathematical, Information, and Computational Sciences of the U.S. Department of Energy under contract number DE-AC02-05CH11231 and by the U.S. Department of Energy under the grant DE-FG-08ER25841. The first two authors also benefited from resources from the Minnesota Supercomputing Institute.

${ }^{\dagger}$ Computational Research Division, Lawrence Berkeley National Laboratory, Berkeley, CA 94720 (eugene.vecharynski@gmail.com).

$\ddagger$ Department of Computer Science and Engineering, University of Minnesota, 200 Union Street S.E., Minneapolis, MN 55455, USA (saad@cs.umn.edu).

$\S$ Department of Modeling, Simulation and Visualization Engineering, Old Dominion University, Norfolk, VA 23529 (msosonki@odu.edu). 
preconditioned matrix, while maintaining a good load balance. The new strategy is tested on several linear systems that arise from discretizations of partial differential equations (PDE's) with strongly varying coefficients.

The choice of the nonoverlapping AS, which is a form of block-diagonal, or block Jacobi, preconditioning, is motivated by several factors. First, the procedure represents the simplest Domain Decomposition (DD) type preconditioner, which is theoretically well-understood and is often of practical interest due to its high degree of parallelism. Second, block-diagonal preconditioning constitutes an important element of a number of more powerful preconditioning schemes, e.g., overlapping or multilevel Schwarz methods, substructuring type algorithms, etc. Our expectation is that the partitions that improve the quality of the nonoverlapping AS preconditioners are also capable of increasing the robustness of these preconditioning schemes.

The problem of partitioning a linear system $A x=b$ is commonly formulated in terms of the adjacency graph $G(A)=(V, E)$ of the coefficient matrix $A=\left(a_{i j}\right)$. Here, $V=\{1,2, \ldots, n\}$ is the set of vertices (nodes) corresponding to the equations/unknowns of the system, and $E$ is the set of edges $(i, j)$, where $(i, j) \in E$ iff $a_{i j} \neq 0$. Throughout, we assume that $A$ is SPD, i.e., $A=A^{*} \succ 0$, which, in particular, implies that the graph $G(A)$ is undirected.

The standard goal of graph partitioning is to partition $G(A)$ into $s$ subgraphs $G_{k}=\left(V_{k}, E_{k}\right)$, where $V_{k} \subseteq V$ and $E_{k} \subseteq E$, such that

$$
\bigcup_{k=1, s} V_{k}=V, \quad \bigcap_{k=1, s} V_{k}=\emptyset, \quad\left|V_{k}\right| \approx n / s
$$

and the size of the edge cut between $G_{k}$ (i.e., the size of the set of edges whose end points are in different $G_{k}$ ) is minimized. Equations and unknowns with numbers in $V_{k}$ are then typically mapped to the same processor; $s$ corresponds to the total number of processors. The requirement on the small edge cut aims at reducing the cost of communications related to the parallel matrix-vector multiplication. The condition $\left|V_{k}\right| \approx n / s$ ensures load balancing. We note that there are alternative models for graph partitioning, based, e.g., on bipartite graphs [14] or hypergraphs [2]. We do not consider these models in the present paper.

The graph partitioning problem is NP-complete. However, there exist a variety of heuristics for solving the problem; see, e.g., [8, 12, 22, 26, 29]. Efficient implementations of partitioning routines are often based on multilevel algorithms, e.g., [17, 20], and are made available in a number of graph partitioning software packages, such as Chaco [15], JOSTLE [38], MeTiS [19], SCOTCH [28], etc.

If the preconditioner quality becomes an objective of the partitioning, then along with the adjacency graph $G(A)$, it is reasonable to consider weights $w_{i j}$ assigned to the edges $(i, j) \in E$, where $w_{i j}$ 's are determined by the coefficients of the matrix $A$. The corresponding algorithm should then be able to take these weights into account and properly use them to perform graph partitioning. An example of such an algorithm has been discussed in [32].

Indeed, one may consider partitioning as part of the pre-processing phase of the preconditioner set up. Then the use of the coefficients of $A$ at the partitioning step, e.g., through the weights $w_{i j}$, represents a natural option. This approach, however, faces a number of issues. For example, given a preconditioning strategy, how does one assign the weights? What are the proper partitioning objectives? How can the partitioning be performed in practice? 
In the present work, these questions are addressed for the case of SPD linear systems and nonoverlapping AS preconditioners. Our rationale is to relate partitioning to the results of the convergence theory for the Preconditioned Conjugate Gradient (PCG) method with block-diagonal preconditioning. More specifically, we regard bipartitioning (i.e., partitioning of the graph into two parts) as an optimization problem that aims at minimizing an upper bound on the condition number of the preconditioned matrix over all possible balanced bipartitions. As a result, we derive a recursive bisection procedure that is built upon a simple weighting scheme and a modification of the standard partitioning objective.

A straightforward approach for dealing with the partitioning problem of this paper would be to assign edge weights as the magnitudes of the corresponding matrix entries and apply a state-of-the-art partitioning algorithm. While this heuristic indeed often improves convergence, it can be substantially outperformed by the new partitioning strategy as will be demonstrated in our numerical experiments.

The presented algorithm relies on the computation of eigenvectors corresponding to the smallest eigenvalues of a generalized eigenvalue problem, which simultaneously involves weighted and standard graph Laplacians. As such, the new strategy is a form of the spectral recursive bisection (RSB) based on a "nonstandard" eigenvalue problem.

Spectral graph partitioning has roots in the works of Fiedler $[9,10]$ and Donath and Hoffman [6, 7]. It is extensively used in many applications, including VLSI circuit design, data clustering, image segmentation; see, e.g., [4, 24, 33] and the references therein. In the scientific computing community, the spectral partitioning was popularized by Pothen et al. in [29] and further studied, e.g., in [16, 34, 37].

Spectral graph partitioning is typically characterized by a good quality of the resulting partitions. This can be attributed to the fact that spectral algorithms utilize global information in contrast to combinatorial algorithms that typically rely on local information. The common criticism of spectral partitioning is its large computational cost, due to the eigenvalue calculations.

Although efficient solution of a specific eigenvalue problem is not the focus of this work, we favor the use of preconditioned eigensolvers. An attractive feature of this approach is that it splits the computation into preconditioning phase and outer iterations, which are based on the matrix-vector multiplications. Ideally, one can expect that the preconditioning scheme encapsulates multilevel strategies (e.g., graph coarsening, uncoarsening, refinement) inherent to the available combinatorial graph partitioners, combined with only a few outer iterations. In our numerical experiments, however, for demonstration purposes, we use Incomplete Cholesky (IC) preconditioning.

The partitioning problem addressed in this paper is closely related to the more traditional task of constructing block-diagonal preconditioners. Indeed, the latter also admits graph formulations and relies on the use of the matrix coefficients; see, e.g., [11]. The main difference, however, lies in the fact that, along with improving the preconditioning quality, the presented partitioning scheme imposes the additional load balance constraint, which is not required for the conventional block-diagonal preconditioning.

The paper is organized as following. In Section 2, we briefly review several known results concerning the block-diagonal preconditioning for SPD matrices. These results motivate the new partitioning scheme, introduced in Section 3. In Section 4, we report on a few numerical examples, where the presented approach is compared to a stateof-the-art partitioning algorithm, MeTiS, with different weighting schemes. 
2. Block-diagonal preconditioning. Consider a block 2-by-2 matrix

$$
A=\left(\begin{array}{cc}
A_{11} & A_{12} \\
A_{12}^{*} & A_{22}
\end{array}\right)
$$

where the diagonal blocks $A_{11}$ and $A_{22}$ are square of size $m$ and $(n-m)$, respectively; the off-diagonal block $A_{12}$ is $m$-by- $(n-m)$. Let $T$ be a block-diagonal preconditioner,

$$
T=\left(\begin{array}{cc}
T_{1} & 0 \\
0 & T_{2}
\end{array}\right)
$$

where $T_{j}=T_{j}^{*} \succ 0, j=1,2$. The dimensions of $T_{1}$ and $T_{2}$ are same as those of $A_{11}$ and $A_{22}$, respectively.

Since both $A$ and $T$ are SPD, the convergence of an iterative method for $A x=b$, such as PCG, is fully determined by the spectrum of the preconditioned matrix $T^{-1} A$. If no information on the exact location of eigenvalues of $T^{-1} A$ is available, then the worst-case convergence behavior of PCG is traditionally described in terms of the condition number $\kappa\left(T^{-1} A\right)$, where $\kappa\left(T^{-1} A\right)=\lambda_{\max }\left(T^{-1} A\right) / \lambda_{\min }\left(T^{-1} A\right)$ with $\lambda_{\max }\left(T^{-1} A\right)$ and $\lambda_{\min }\left(T^{-1} A\right)$ denoting the largest and the smallest eigenvalues of the preconditioned matrix, respectively. The question which arises is how we can bound $\kappa\left(T^{-1} A\right)$ for an arbitrary $A$ and a block-diagonal $T$. The answer to this question is given, e.g., in [1, Chapter 9]. Below, we briefly state the main result.

DeFinition 2.1. Let $U_{1}$ and $U_{2}$ be finite dimensional spaces, such that $A$ in (2.1) is partitioned consistently with $U_{1}$ and $U_{2}$. The constant

$$
\gamma=\max _{w_{1} \in W_{1}, w_{2} \in W_{2}} \frac{\left|\left(w_{1}, A w_{2}\right)\right|}{\left(w_{1}, A w_{1}\right)^{1 / 2}\left(w_{2}, A w_{2}\right)^{1 / 2}},
$$

where $W_{1}$ and $W_{2}$ are subspaces of the form

$$
W_{1}=\left\{u=\left(\begin{array}{c}
u_{1} \\
\boldsymbol{O}
\end{array}\right), u_{1} \in U_{1}\right\}, W_{2}=\left\{u=\left(\begin{array}{c}
\boldsymbol{0} \\
u_{2}
\end{array}\right), u_{2} \in U_{2}\right\}
$$

is called the Cauchy-Bunyakowski-Schwarz (CBS) constant.

In (2.3), $(u, v)=v^{*} u$ denotes the standard inner product. We note that $\gamma$ can be interpreted as a cosine of an angle between subspaces $W_{1}$ and $W_{2}$. Thus, since, additionally, $W_{1} \cap W_{2}=\left(\begin{array}{l}\mathbf{0} \mathbf{0}\end{array}\right)^{*}$, it is readily seen that $0 \leq \gamma<1$. Also we note that $\gamma$ is the smallest possible constant satisfying the strengthened Cauchy-SchwarzBunyakowski inequality $\left|\left(w_{1}, A w_{2}\right)\right| \leq \gamma\left(w_{1}, A w_{1}\right)^{1 / 2}\left(w_{2}, A w_{2}\right)^{1 / 2}$, which motivates its name.

Theorem 2.2 ([1], Chapter 9). If $T_{1}=A_{11}$ and $T_{2}=A_{22}$ in (2.2), and $A$ in (2.1) is $S P D$, then $\kappa\left(T^{-1} A\right) \leq(1+\gamma) /(1-\gamma)$.

The bound given by Theorem 2.2 is sharp. In the subsequent sections, we use this result to derive a new partitioning algorithm.

3. Partitioning using matrix coefficients. Given decomposition $\left\{V_{k}\right\}_{k=1}^{s}$ of the set $V=\{1,2, \ldots, n\}$ (possibly with overlapping $V_{k}$ ), we consider the AS preconditioning for an SPD system $A x=b$. The preconditioning procedure is given in Algorithm 3.1. By $A\left(V_{l}, V_{k}\right)$ we denote a submatrix of $A$ located at the intersection of rows with indices in $V_{l}$ and columns with indices in $V_{k}$. Similarly, $r\left(V_{k}\right)$ denotes the subvector of $r$, containing entries from positions $V_{k}$. In this section, we focus on the case where sets (subdomains) $\left\{V_{k}\right\}_{k=1}^{s}$ are nonoverlapping, i.e., (1.1) holds. This means that Algorithm 3.1 gives a nonoverlapping AS preconditioner.

Algorithm 3.1 (AS preconditioner). Input: $A, r,\left\{V_{k}\right\}_{k=1}^{s}$. Output: $w=T^{-1} r$. 
1. For $k=1, \ldots, s, D o$

2. Set $A_{k}:=A\left(V_{k}, V_{k}\right), r_{k}:=r\left(V_{k}\right)$, and $w_{k}=\boldsymbol{O} \in \mathbb{R}^{n}$.

3. Solve $A_{k} \delta=r_{k}$.

4. $\quad$ Set $w_{k}\left(V_{k}\right):=\delta$.

5. EndDo

6. $w=w_{1}+\ldots+w_{s}$.

Let $P$ be a permutation matrix which corresponds to the reordering of $V$ according to the partition $\left\{V_{k}\right\}_{k=1}^{s}$, where the elements in $V_{1}$ are labeled first, in $V_{2}$ second, etc. Then the AS preconditioner $T$, given by Algorithm 3.1, can be written in the matrix form as $T=P^{T} \bar{T} P$, where $\bar{T}=$ blockdiag $\left\{A_{1} \ldots, A_{s}\right\}$ and $A_{k}=A\left(V_{k}, V_{k}\right)$. Thus, Algorithm 3.1 results in the block-diagonal, or block Jacobi, preconditioner, up to a permutation of its rows and columns.

3.1. Optimal bipartitions. Let $s=2$, so that (1.1) corresponds to a bipartition

$$
V=I \cup J, \quad I \cap J=\emptyset, \quad|I|=|J|=n / 2 .
$$

Here, we assume that $n$ is even. This guarantees the existence of fully balanced bipartitions, such that vertex sets $I$ and $J$ are of the same size, $n / 2$. Similarly, we assume that each connected component of $G(A)$ also has an even number of vertices. The above assumptions, however, will not be a restriction for the practical algorithm described below.

The following theorem provides a relation between a given bipartition and $\kappa\left(T^{-1} A\right)$. The theorem is a direct consequence of Theorem 2.2 and is based on the fact that symmetric permutations preserve the spectra.

THEOREM 3.1. Let $\{I, J\}$ in (3.1) be a bipartition of $V$ (possibly unbalanced). Let $T$ be the AS preconditioner for system $A x=b$ with an SPD matrix $A$, given by Algorithm 3.1, with respect to the bipartition $\{I, J\}$. Then,

$$
\kappa\left(T^{-1} A\right) \leq \frac{1+\gamma_{I J}}{1-\gamma_{I J}}
$$

where

$$
\gamma_{I J}=\max _{u \in W_{I}, v \in W_{J}} \frac{|(u, A v)|}{(u, A u)^{1 / 2}(v, A v)^{1 / 2}} .
$$

The spaces $W_{I}$ and $W_{J}$ are the subspaces of $\mathbb{R}^{n}$ with dimensions $m$ and $(n-m)$, respectively, such that

$$
W_{I}=\left\{u \in \mathbb{R}^{n}: u(J)=\boldsymbol{O}\right\}, W_{J}=\left\{v \in \mathbb{R}^{n}: v(I)=\boldsymbol{O}\right\} .
$$

Proof. For the given bipartition $\{I, J\}$ in (3.1), the preconditioner $T$, constructed by Algorithm 3.1, is of the form

$$
T=P^{T} B P, B=\left(\begin{array}{cc}
A_{I} & 0 \\
0 & A_{J}
\end{array}\right),
$$

where $A_{I}=A(I, I), A_{J}=A(J, J)$, and $P$ is a permutation matrix corresponding to the reordering of $V$ with respect to the partition $\{I, J\}$. In particular, for any $x$, the vector $y=P x$ is such that $y=(x(I) x(J))^{T}$, i.e., the entries of $x$ with indices in $I$ 
become the first $m$ components of $y$, while the entries with indices in $J$ get positions from $m+1$ through $n$.

We observe that the condition number of the matrix $T^{-1} A$ is the same as the condition number of the matrix $B^{-1} C$, where $C=P A P^{T}$ and $B$ in (3.5). Indeed, since a unitary similarity transformation

$$
P\left(T^{-1} A\right) P^{T}=P\left(P^{T} B^{-1} P A\right) P^{T}=B^{-1}\left(P A P^{T}\right)=B^{-1} C,
$$

preserves the eigenvalues of $T^{-1} A$, we have $\kappa\left(T^{-1} A\right)=\kappa\left(B^{-1} C\right)$, where $\kappa(\cdot)=$ $\lambda_{\max }(\cdot) / \lambda_{\min }(\cdot)$.

The matrix $C$ represents a symmetric permutation of $A$ with respect to the given bipartition $\{I, J\}$, and, thus, can be written in the 2-by-2 block form,

$$
C=P A P^{T}=\left(\begin{array}{cc}
A_{I} & A_{I J} \\
A_{I J}^{*} & A_{J}
\end{array}\right)
$$

where $A_{I}=A(I, I), A_{J}=A(J, J)$, and $A_{I J}=A(I, J)$. Since $C$ is SPD and the preconditioner $B$ in (3.5) is the block diagonal of $C$, we apply Theorem 2.2 to get the upper bound on the condition number $\kappa\left(B^{-1} C\right)$, and hence bound (3.2) on $\kappa\left(T^{-1} A\right)$, where, according to Definition 2.1, the CBS constant $\gamma \equiv \gamma_{I J}$ is given by

$$
\begin{aligned}
\gamma_{I J} & =\max _{w_{1} \in W_{1}, w_{2} \in W_{2}} \frac{\left|\left(w_{1}, C w_{2}\right)\right|}{\left(w_{1}, C w_{1}\right)^{1 / 2}\left(w_{2}, C w_{2}\right)^{1 / 2}} \\
& =\max _{w_{1} \in W_{1}, w_{2} \in W_{2}} \frac{\left|\left(w_{1}, P A P^{T} w_{2}\right)\right|}{\left(w_{1}, P A P^{T} w_{1}\right)^{1 / 2}\left(w_{2}, P A P^{T} w_{2}\right)^{1 / 2}} \\
& =\max _{w_{1} \in W_{1}, w_{2} \in W_{2}} \frac{\left|\left(P^{T} w_{1}, A P^{T} w_{2}\right)\right|}{\left(P^{T} w_{1}, A P^{T} w_{1}\right)^{1 / 2}\left(P^{T} w_{2}, A P^{T} w_{2}\right)^{1 / 2}} .
\end{aligned}
$$

The matrix $P^{T}$ defines the permutation that is the "reverse" of the one corresponding to $P$. Thus, the substitution $u=P^{T} w_{1}$ and $v=P^{T} w_{2}$ leads to expression (3.3)-(3.4) for $\gamma_{I J}$, where the $W_{I}$ and $W_{J}$ contain vectors, which can have nonzero entries only at positions defined by $I$ or $J$, respectively.

The sharp upper bound (3.2) represents a meaningful indicator of the preconditioner quality. Thus, as an optimal bipartition, we can choose $\{I, J\}$, such that $\left(1+\gamma_{I J}\right) /\left(1-\gamma_{I J}\right)$ or, equivalently, the CBS constant $\gamma_{I J}$, is minimized. More precisely, we define an optimal bipartition $\left\{I_{o p t}, J_{o p t}\right\}$ to be such that

$$
\left\{I_{\text {opt }}, J_{\text {opt }}\right\}=\underset{\substack{I, J \subset V=\{1, \ldots, n\},|I|=|J|=\frac{n}{2}, J=V \backslash I}}{\operatorname{argmin}} \gamma_{I J},
$$

where $\gamma_{I J}$ is defined in (3.3).

A straightforward solution of optimization problem (3.7) entails evaluating (3.3) for a very large, namely $n ! /[(n / 2) !]^{2}$, possible choices of the partitions $\{I, J\}$. While this indicates that the problem is likely to be NP-hard, it is not clear whether an efficient solution to (3.7) can be found.

Therefore, our idea is to replace (3.7) by a related simpler problem, such that the minimizer of the latter approximates (in terms of smallness of the CBS constant) $\left\{I_{\text {opt }}, J_{\text {opt }}\right\}$ rather than determines it exactly. Below, we discuss several approaches. 
3.2. The minimal averaged cut. In order to obtain a simpler optimization problem, let us replace $\gamma_{I J}$ in (3.7) by some $\tilde{\gamma}_{I J}$, such that $\tilde{\gamma}_{I J}$ captures information on $\gamma_{I J}$ and is easy to compute. We define $\tilde{\gamma}_{I J}$ as following.

Given $\{I, J\}$, instead of maximizing the ratio

$$
\frac{|(u, A v)|}{(u, A u)^{1 / 2}(v, A v)^{1 / 2}},
$$

as required for computing $\gamma_{I J}$ in (3.3), we introduce a set of pairs

$$
S=\left\{\left(e_{i}, e_{j}\right): i \in I, j \in J, a_{i j} \neq 0\right\} \subset W_{I} \times W_{J},
$$

where $e_{k} \in \mathbb{R}^{n}$ denotes the unit vector with 1 at position $k$, and calculate (3.8) on all $\left(e_{i}, e_{j}\right) \in S$. Note that the cardinality of $S$ is equal to the size of the cut between $I$ and $J$, further denoted by $\operatorname{cut}(I, J)$. The resulting values are averaged. This gives a constant $\tilde{\gamma}_{I J}$, such that

$$
\tilde{\gamma}_{I J}=\frac{w(I, J)}{\operatorname{cut}(I, J)},
$$

where $w(I, J)=\sum_{i \in I, j \in J} w_{i j}, w_{i j}=\left|a_{i j}\right| / \sqrt{a_{i i} a_{j j}} \in(0,1)$.

The constant $\tilde{\gamma}_{I J}$ has a transparent meaning in terms of the adjacency graph $G(A)$. Assigning the weights $w_{i j}$ to the edges $(i, j),(3.10)$ can be interpreted as a ratio of the cut weight, $w(I, J)$, to the cut size; or, equivalently, as the averaged cut weight. At the same time, $\tilde{\gamma}_{I J}$ is closely related to $\gamma_{I J}$. In particular, $\tilde{\gamma}_{I J} \leq \gamma_{I J}$ for all $\{I, J\}$. Thus, we replace optimization problem (3.7) by

$$
\left\{\tilde{I}_{o p t}, \tilde{J}_{o p t}\right\}=\underset{\substack{I, J \subset V=\{1, \ldots, n\} \\|I|=|J|=\frac{n}{2}, J=V \backslash I}}{\operatorname{argmin}} \tilde{\gamma}_{I J} .
$$

The minimizer $\left\{\tilde{I}_{o p t}, \tilde{J}_{\text {opt }}\right\}$ is expected to approximate $\left\{I_{o p t}, J_{o p t}\right\}$. Formally, (3.10)(3.11) represents the problem of graph bipartitioning, where the targeted cut has the smallest, in average, weight. We call such a cut the minimal averaged cut (Acut).

Minimization of objective (3.10) is achieved by bipartitions that provide a balance between the two concurrent requirements on minimizing the cut weight and maximizing the number of edges included in the cut. Thus, (3.10)-(3.11) gives cuts that include a relatively large number of "light-weighted" edges. As shown in our numerical experiments, for a number of systems arising from discretizations of partial differential equations with strongly varying coefficients, this allows us to recognize the boundaries of subregions that correspond to different coefficient magnitudes. The resulting subdomains are experimentally shown to improve preconditioning quality.

3.3. Spectral Acut computations. Optimization problem (3.11) is still hard to solve exactly since it encompasses the original graph partitioning problem which is known to be NP-complete [35]. Therefore, we address below the question of approximating Acut for practical applications. We propose a spectral bipartitioning technique.

Let $p$ denote the indicator vector of size $n$, with the components defined as

$$
p(k)=\left\{\begin{array}{c}
1, k \in I, \\
-1, k \in J .
\end{array}\right.
$$


Then, for a given $\{I, J\}$,

$$
\begin{aligned}
4 w(I, J) & =\sum_{(i, j) \in E} w_{i j}(p(i)-p(j))^{2}=\sum_{(i, j) \in E} w_{i j}\left(p(i)^{2}+p(j)^{2}\right)-2 \sum_{(i, j) \in E} w_{i j} p(i) p(j) \\
& =\sum_{i=1}^{n} d_{w}(i) p(i)^{2}-\sum_{i, j=1}^{n} w_{i j} p(i) p(j)
\end{aligned}
$$

where $d_{w}(i)=\sum_{j \in N(i)} w_{i j}$ is the weighted degree of the vertex $i ; N(i)$ denotes the vertices adjacent to $i$. Thus, $w(I, J)$ can be written as a bilinear form,

$$
4 w(I, J)=p^{T} L_{w} p, \quad L_{w}=D_{w}-W,
$$

where $D_{w}=\operatorname{diag}\left(d_{w}(1), \ldots, d_{w}(n)\right)$ is the weighted degree matrix and $W=\left(w_{i j}\right)$ denotes the weighted adjacency matrix. Similarly, setting $w_{i j}=1$ for all edges $(i, j)$ of $G(A)$, we get the expression for $\operatorname{cut}(I, J)$,

$$
4 \operatorname{cut}(I, J)=p^{T} L p, \quad L=D-Q,
$$

where $D$ is the degree matrix and $Q=\left(q_{i j}\right)$ is the adjacency matrix. The matrices $L_{w}$ and $L$ denote the weighted and unweighted graph Laplacians. Thus, given $\{I, J\},(3.13)$ and (3.14) allow representing (3.10) as a ratio of two bilinear forms, i.e., $\tilde{\gamma}_{I J}=p^{T} L_{w} p / p^{T} L p$.

Let us assume that $G(A)$ has $q \geq 1$ connected components $\left(V_{l}, E_{l}\right)$, where $\left|V_{l}\right|$ are even. We introduce vectors $z_{1}, \ldots, z_{q}$, such that

$$
z_{l}(k)=\left\{\begin{array}{l}
1, k \in V_{l} \\
0, k \notin V_{l}
\end{array}\right.
$$

i.e., the entries of $z_{l}$ corresponding to vertices in $V_{l}$ are 1 , and 0 elsewhere. Note that if $q=1$, then we obtain a single vector of ones.

Problem (3.11) can now be written as

$$
\tilde{p}_{\text {opt }}=\underset{p}{\operatorname{argmin}} \frac{p^{T} L_{w} p}{p^{T} L p}, \quad p^{T} z_{l}=0, \quad l=1, \ldots, q,
$$

where the minimizer $\tilde{p}_{\text {opt }}$ is searched over all feasible indicator vectors. The condition $p^{T} z_{l}=0$ ensures that all the connected components are bipartitioned into two equalsized sets of vertices. Hence, $|I|=|J|=n / 2$, as required.

In order to approximate solution of (3.16), we relax the problem by embedding it into the real space. More specifically, we consider the minimization

$$
\min _{v \in \mathbb{R}^{n}} \frac{v^{T} L_{w} v}{v^{T} L v}, \quad v \in \operatorname{span}\left\{z_{1}, \ldots, z_{q}\right\}^{\perp},
$$

of the generalized Rayleigh quotient on the orthogonal complement of the subspace spanned by vectors $z_{l}$ in (3.15). We expect the minimizer of (3.17) to provide an approximation to the optimal indicator vector $\tilde{p}_{\text {opt }}$ from (3.16).

Both $L_{w}$ and $L$ are symmetric positive semi-definite, with the dimension of the nullspace equal to the number of connected components of $G(A)$. In particular,

$$
\operatorname{null}\left(L_{w}\right)=\operatorname{null}(L)=\operatorname{span}\left\{z_{1}, \ldots, z_{q}\right\} .
$$


Thus, $v^{T} L_{w} v / v^{T} L v$ in (3.17) is minimized on the orthogonal complement of the nullspace of the two matrices, where both $L$ and $L_{w}$ are SPD. This implies that the minimum in (3.17) exists. It is achieved on the eigenvector associated with smallest eigenvalue of the symmetric generalized eigenvalue problem

$$
L_{w} v=\lambda L v, \quad v \in \operatorname{span}\left\{z_{1}, \ldots, z_{q}\right\}^{\perp} .
$$

Solution of (3.18) can be viewed as an analogue of the Fiedler vector $[9,10]$. The bipartition is formed by assigning the indices of its $\lceil n / 2\rceil$ smallest components to $I$ and the rest to $J$. As shown in our numerical experiments, the eigenvector of (3.18) may deliver disconnected subdomains, even though the original graph is connected. Note that the assumption on the even sizes of the vertex sets of $G(A)$ and its connected components is not restrictive any more, and is skipped for relaxed problem (3.18).

Finally, let us observe that if the weights $w_{i j}$ are the same for all edges, then the graph Laplacians $L_{w}$ and $L$ represent multiples of each other. In this case, the solution of (3.18) is given by multiple orthogonal eigenvectors that correspond to the only nonzero eigenvalue of multiplicity $n-q$, i.e., the result of spectral Acut computations is highly uncertain. Such a situation is an indicator of the fact that the coefficient matrix $A$ has extremely regular behavior of its entries. Therefore, if all $w_{i j}$ are the same, or only slightly different, we suggest using standard partitioning criteria.

3.4. The minimal weighted cut. We now relate the problem of minimizing the CBS constant to the standard objective for graph partitioning. In particular, we consider replacing (3.7) by minimization of the cut weight under the load balance constraint.

Given a bipartition $\{I, J\}$ in (3.1), similarly to (3.9), we define the set

$$
\bar{S}=\left\{\left(e_{i}, e_{j}\right): i \in I, j \in J\right\} \subset W_{I} \times W_{J} .
$$

Unlike (3.9), (3.19) contains all pairs $\left(e_{i}, e_{j}\right)$ with $i \in I$ and $j \in J$, including those that correspond to $a_{i j}=0$. Instead of maximizing (3.8), as required to compute $\gamma_{I J}$, we evaluate (3.8) on all $(n / 2)^{2}$ pairs in (3.19) and then find the average. Thus, for a given $\{I, J\}$ we define a constant $\bar{\gamma}_{I J} \leq \gamma_{I J}$, such that

$$
\bar{\gamma}_{I J}=\frac{4}{n^{2}} w(I, J),
$$

where $w(I, J)$ is the cut weight, defined as in (3.10).

Following the pattern of the previous subsections, instead of (3.7), we suggest solving optimization problem

$$
\left\{\bar{I}_{\text {opt }}, \bar{J}_{\text {opt }}\right\}=\underset{\substack{I, J \subset V=\{1, \ldots, n\},|I|=|J|=\frac{n}{2}, J=V \backslash I}}{\operatorname{argmin}} \bar{\gamma}_{I J},
$$

where $\left\{\bar{I}_{o p t}, \bar{J}_{o p t}\right\}$ is expected to approximate an optimal $\left\{I_{o p t}, J_{o p t}\right\}$ in (3.7). It is readily seen that (3.20)-(3.21) delivers the well-known problem of graph partitioning, which aims at finding equal-sized vertex sets $I$ and $J$ with the minimal cut weight. The solution of (3.20)-(3.21) can be approximated by an available graph partitioning scheme which admits edge weighting. In our numerical examples, we use MeTiS.

The principal difference between (3.10)-(3.11) and (3.20)-(3.21) is in that (3.20)(3.21) delivers cuts of a cumulatively small weight, which may generally contain both 
"heavy-weighted" and "light-weighted" edges. At the same time, cuts given by (3.10)(3.11) favor edges with small weights. For example if $A$ is given by a PDE with jumps in coefficients, such edges connect subdomains corresponding to different coefficient magnitudes, whereas edges with large $w_{i j}$ are in the interior of these subdomains. Therefore, unless forced by the load balance constraint, (3.10)-(3.11) excludes the undesirable possibility of "cutting" inside jump subregions.

3.5. Acut by recursive spectral bisection. Let $\{I, J\}$ be given by Acut described in Subsection 3.3. A natural way to construct further partitions (1.1) is to apply the bipartitioning process separately for subgraphs of $G(A)$ corresponding to $I$ and $J$, then to the resulting partitions and so on, until all the subpartitions are sufficiently small. We summarize this in the following algorithm.

Algorithm 3.2 (Acut-RSB $(A)$ ). Input: A. Output: Partition $\left\{V_{i}\right\}$.

1. Form $G(A)$. Assign weights $w_{i j}=\left|a_{i j}\right| / \sqrt{a_{i i} a_{j j}}$.

2. Construct $L_{w}=D_{w}-W$ and $L=D-Q$.

3. Find connected components $\left\{E_{l}, V_{l}\right\}$. Define $z_{l}$ in (3.15).

4. Find the eigenvector associated with the smallest eigenvalue of (3.18).

5. Define $\{I, J\}$ based on the computed eigenvector.

6. Apply the algorithm recursively:

If $|I|>$ maxSize then call Acut-RSB $(A(I, I))$, else return $I$.

If $|J|>\operatorname{maxSize}$ then call Acut-RSB $(A(J, J))$, else return $J$.

The parameter maxSize in Algorithm 3.2 is provided by the user, and should be chosen to ensure that $\left|V_{k}\right| \approx n / s$. The connected components in Step 3 can be detected by standard algorithms based on the breadth-first search or the depth-first search [3]. Note that weights $w_{i j}$ are the same on all levels of the recursion. In practice, they are assigned only once, on the top level.

Clearly, if eigenvalue problem (3.18) in step 4 is replaced by computing the Fiedler vector of either $L_{w} v=\lambda v$ or $L v=\lambda v$, then Algorithm 3.2 reduces to the well-known RSB scheme; see, e.g., [30]. We suggest that before the recursive Acut-RSB calls in Step 6 , one checks if $A(I, I)$ and $A(J, J)$ indeed have large variations in magnitudes of their entries, e.g., by assessing the variance of the coefficients. If the entries of $A(I, I)$ or $A(J, J)$ exhibit a regular behavior, we recommend invoking one of the standard, unweighted, partitioning algorithms.

4. Numerical results. In this section, we apply Acut-RSB to several SPD test problems and compare the partitioning results to more traditional approaches delivered by the MeTiS algorithm with various weighting schemes. For each test problem, we use the partitions produced by all algorithms to construct the AS preconditioners, which are then supplied to the PCG iteration. We further refer to this solution scheme as PCG-AS.

In our examples, we consider three types of MeTiS partitions. The first type results from applying the algorithm to the unweighted adjacency graph, so that the coefficient information is completely skipped. In contrast, the other two types are obtained by running MeTiS for the weighted graphs, where the weights are based on the matrix coefficients. In particular, we use weighting schemes $y=\left\{y_{i j}\right\}$ and $t=\left\{t_{i j}\right\}$, with the edge weights $y_{i j}=\left\lceil\gamma\left|a_{i j}\right| / \sqrt{a_{i i} a_{j j}}\right\rceil$ and $t_{i j}=\left\lceil\delta\left|a_{i j}\right|\right\rceil$. Here, the parameter $\gamma$ is a large integer and $\delta>0$. We denote the corresponding partitioning methods by $\operatorname{MeTiS}(y)$ and $\operatorname{MeTiS}(t)$, respectively. Note that a closely related weighting scheme 
has been used in [13].

Let us remark that $y_{i j}$ can be written as $y_{i j}=\left\lceil\gamma w_{i j}\right\rceil$, where $w_{i j}$ are the weights in the CBS constant based partitioning formulations (3.10)-(3.11) and (3.20)-(3.21). Thus, whereas Acut-RSB targets (3.10)-(3.11), MeTiS $(y)$ is expected to approximate partitions in (3.20)-(3.21). The constant $\gamma$ and the "ceil" operation in the definition of $y_{i j}$ are introduced to satisfy the MeTiS requirement on the integer weight values (recall that $w_{i j} \in(0,1)$ ).

In our numerical experiments, we have observed that $\operatorname{MeTiS}(y)$ gives better results than the RSB scheme based on the Fiedler vector for $L_{w} v=\lambda v$, which approximates solution of (3.20)-(3.21). Therefore, the latter is not reported in the examples below.

The choice of $t_{i j}=\left\lceil\delta\left|a_{i j}\right|\right\rceil$ for $\operatorname{MeTiS}(t)$ is motivated by the computational experience, suggesting that each edge should be weighted with (essentially) the absolute value of the corresponding matrix entry. While straightforward and well-known among practitioners, we were not able to find direct references to such a weighting scheme in the literature.

In our tests, we define $t_{i j}$ with $\delta$ from $(0,1]$. This choice of $\delta$ "damps" the magnitudes of $\left|a_{i j}\right|$. It ensures that the weighted cut sizes computed by $\operatorname{MeTiS}(t)$ do not exceed the upper limit of the integer data type size, in which case the behavior of the algorithm can be unpredictable. Similar to $y_{i j}$, the "ceil" operation is introduced to maintain integer weights. Note that if $A$ is diagonally scaled, $y_{i j}$ and $t_{i j}$ coincide.

Throughout, the reported partitioning results produced by $\operatorname{MeTiS}(y)$ and $\operatorname{MeTiS}(t)$ correspond to the best values of $\gamma$ and $\delta$ observed during our experiments. In all cases, MeTiS has been invoked with the "PartGraphRecursive" option, which enables the recursive bipartitioning [21] and guarantees the strict load balance. Note that all our experiments are performed in MATLAB; the MeTiS library is accessed through the MEX interface.

Unlike MeTiS, which represents a combinatorial partitioning technique, the new Acut-RSB algorithm relies on computing eigenvectors. In all of our tests, as an eigensolver, we use Locally Optimal Block Preconditioned Conjugate Gradient (LOBPCG) method [23]. Our choice has been motivated mainly by the fact that, unlike the Lanczos algorithm [27], LOBPCG can solve generalized eigenvalue problems, such as (3.18), without requiring any factorization of the singular matrices $L_{w}$ and $L$. Additionally, LOBPCG encapsulates preconditioning to accelerate convergence and has a relatively modest storage requirement.

The LOBPCG algorithm is a form of a (block) three-term recurrence, which locally optimizes the Rayleigh quotient; see [23] for more details. Given a suitable SPD preconditioner, the method is known to be efficient for large-scale eigenvalue computations. In our experiments, we construct preconditioners using IC factorization of matrices $L_{w}+\sigma I$ with a drop tolerance of $10^{-3}$. To ensure that the IC procedure is correctly applied to the SPD matrices, the parameter $\sigma$ is assigned a small positive value. In particular, we set $\sigma=0.1$.

In all LOBPCG runs, we choose a random initial guess from $\operatorname{span}\left\{z_{1}, \ldots, z_{q}\right\}^{\perp}$ and at each step project the preconditioned residuals to this subspace, so that the LOBPCG iterations are kept in $\operatorname{span}\left\{z_{1}, \ldots, z_{q}\right\}^{\perp}$. Note that the amount of storage and computations required to orthogonalize against $\operatorname{span}\left\{z_{1}, \ldots, z_{q}\right\}^{\perp}$ does not depend on $q$, i.e., is the same as for the $n$-vector of all ones.

Finally, let us remark that the partitioning runtimes exhibited by MeTiS have been notably lower than those of Acut-RSB. This can be attributed to the spectral nature of the new algorithm. Additionally, the runtime difference has been exacer- 
bated by the fact that, in contrast to the MEX-interfaced MeTiS library calls, our Acut-RSB code is purely in MATLAB.

However, as demonstrated below, despite longer runtimes, Acut-RSB often leads to a significantly smaller number of iterations of the linear solver. In this context, the partitioning cost (i.e., the pre-processing phase of the preconditioner set up) becomes less important. In fact, our goal is precisely this: to get a more reliable iterative solution procedure by paying a higher cost in the preprocessing.

2D diffusion equation. Let us consider the diffusion equation on a unit square,

$$
-\frac{\partial}{\partial x}\left(a(x, y) \frac{\partial u}{\partial x}\right)-\frac{\partial}{\partial y}\left(b(x, y) \frac{\partial u}{\partial y}\right)=f(x, y),(x, y) \in[0,1] \times[0,1],
$$

with zero Dirichlet boundary conditions and the coefficients given by the piecewise constants

$$
a(x, y)=b(x, y)=\left\{\begin{array}{cl}
10^{5}, & 0.25<x, y<0.75 \\
1, & \text { otherwise }
\end{array}\right.
$$

which strongly vary (jump) across the two subdomains; see Figure 4.1.

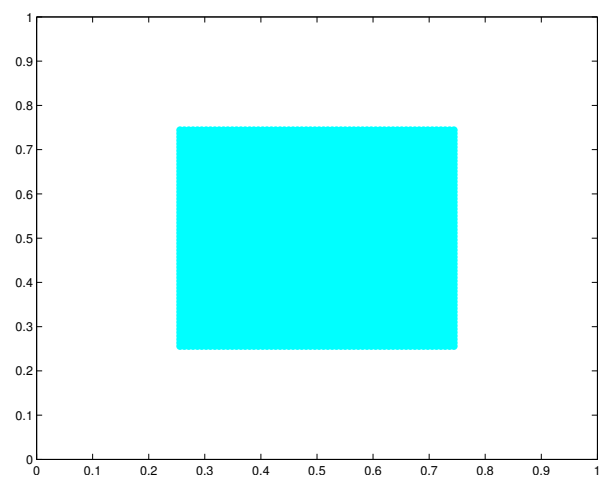

FIG. 4.1. Location of the jump in coefficients (4.2) for problem (4.1). The shaded (cyan for the color plot) subdomain corresponds to the jump region $(0.25,0.75) \times(0.25,0.75)$.

In order to discretize (4.1), we introduce a 128-by-128 (interior points) uniform grid and use the standard 5-point finite difference (FD) stencil. The resulting linear system, $A x=b$, is SPD of size $n=16,384$. For testing purposes, the right-hand side $b$ is randomly chosen. Here, and below, the FD matrices have been generated using the SPARSKIT library [31].

Figure 4.2 shows the bipartitions produced by different methods. It is readily seen that Acut-RSB precisely detects the jump region and places most of the cut along its boundary, without "cutting" inside. In the framework of the DD type methods, the latter is consistent with a common recommendation to include the regions corresponding to different model parameters into separate subdomains. The eigenvector, used to define the bipartition in Acut-RSB, is shown in Figure 4.8 (left). The values of $\gamma$ and $\delta$ for the weights in $\operatorname{MeTiS}(y)$ and $\operatorname{MeTiS}(t)$ have been set to $10^{5}$ and 1 , respectively. The convergence tolerance for LOBPCG is $10^{-7}$.

Similar to Acut-RSB, we observe that the bipartition produced by $\operatorname{MeTiS}(t)$ also recognizes the region of the jump and isolates it in a separate subdomain. The visual difference, however, is in that the boundaries of this subdomain are not as smooth as for the Acut and do not tightly follow the boundaries of the jump region. 

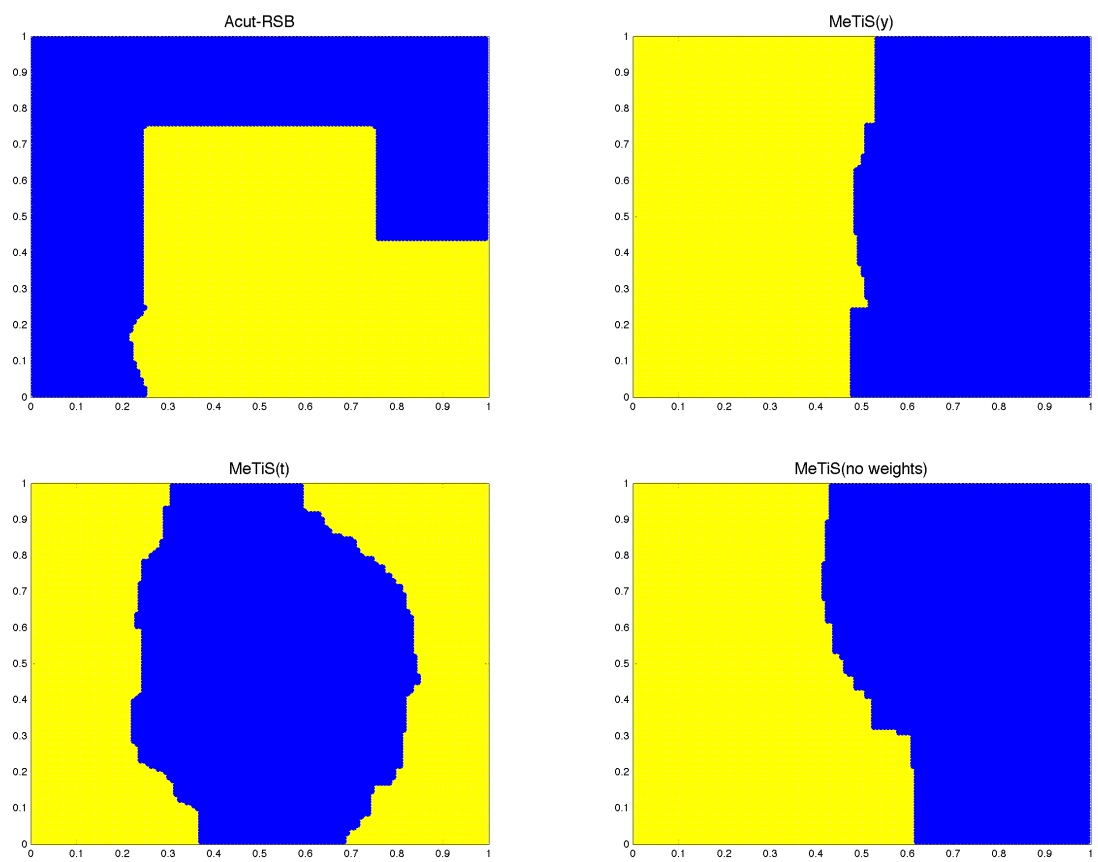

FIG. 4.2. Bipartitions for problem (4.1) with coefficients in (4.2).

In Figure 4.3, we present the results obtained after an additional step of the recursive bipartitioning (with the same $\gamma, \delta$, and the LOBPCG convergence tolerance as above). In this case, the partitioning procedures deliver 4 subdomains. Note that, regardless of the weighting scheme, all the three MeTiS runs perform long cuts within the jump region. In contrast, Acut-RSB essentially "crops" the central square, with the top right corner assigned to a different subdomain merely to ensure the strict load balance.

Since one of the two subdomains resulting from the Acut-RSB bipartioning does not contain a jump region (see Figure 4.2, top left), we use a standard partitioning approach at the recursive step on this subdomain. In particular, we use the spectral bisection based on the Fiedler vector [29].

Throughout all of our experiments, we set the number of targeted subdomains to be small, i.e., at most 16 as in the 3D elasticity example considered below. Due to simplicity of the geometries of the tested problems, convergence of the Acut based solver deteriorates if the number of subdomains is increased. In this case, Acut-RSB begins to partition inside the regions with similar coefficient magnitudes, i.e., it is forced by the load balance constraint to discard "heavy" edges. At some point, this hinders the convergence. Generally, we expect that the number of subdomains, and hence of processors, will depend on the problem geometry and coefficients.

In Figure 4.4, we show the effects of partitioning on the convergence of a preconditioned iterative scheme. In particular, we plot the convergence curves of PCG-AS with the preconditioners defined on different partitions. In both cases, with 2 and 4 subdomains, we observe that PCG-AS based on the Acut-RSB partitioning in Algorithm 3.2 performs a significantly smaller number of iterations. 

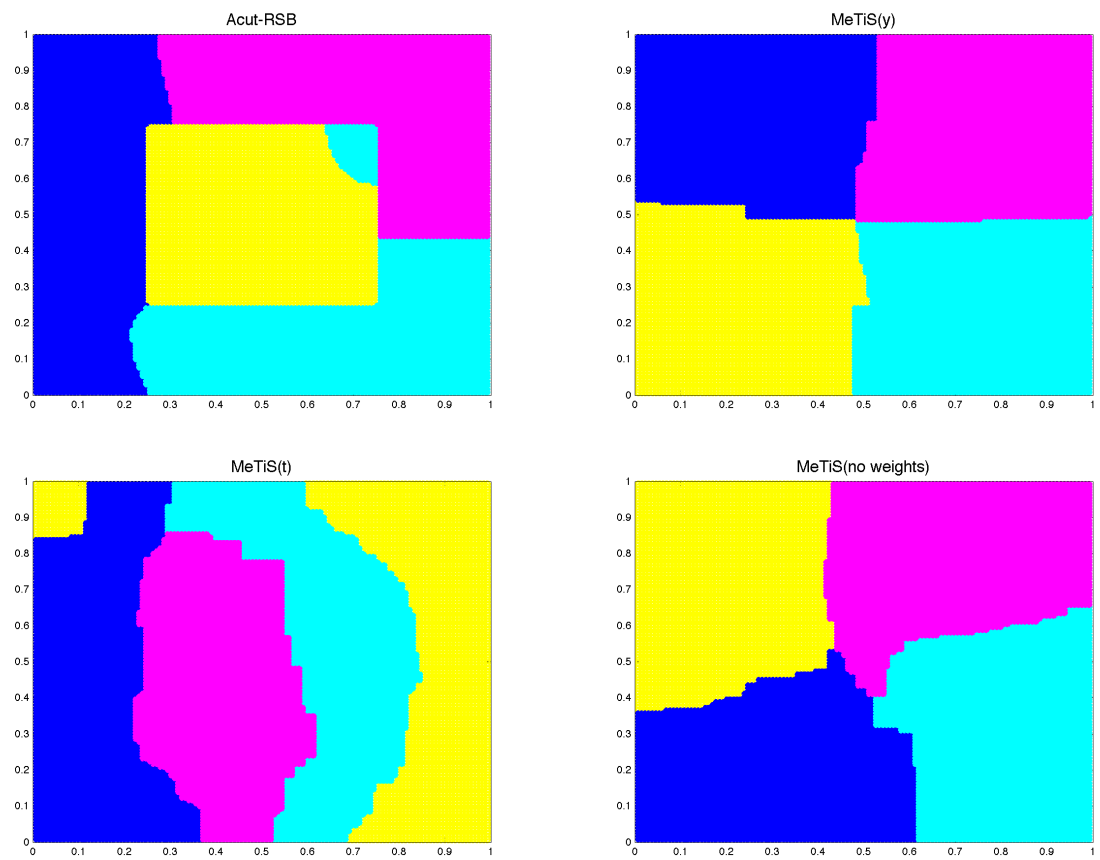

FIG. 4.3. Partitioning into 4 subdomains for problem (4.1) with coefficients in (4.2).
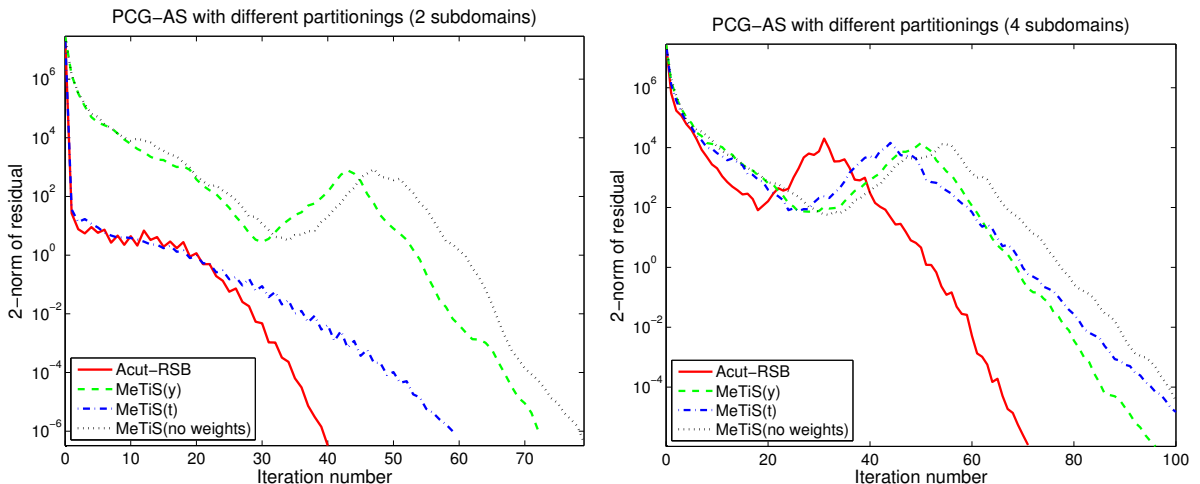

FIG. 4.4. Convergence of $P C G-A S$ with different partitions for problem (4.1) with coefficients in (4.2). The linear system of size $n=16,384$ is partitioned into 2 (left) and 4 (right) subdomains.

In Table 4.1, we show the relative cut sizes and amounts of the coefficient information discarded from the matrix after partitioning to construct the preconditioners. In particular, we report the quantities

$$
\text { relcut }=\frac{\text { cut size }}{\mathrm{nnz}} \times 100 \%, \quad \text { relcoef }=\frac{\sum_{(i, j) \in \mathrm{cut}}\left|a_{i j}\right|}{\sum_{i, j}\left|a_{i j}\right|} \times 100 \%
$$

where "cut size" is the number of edges in the given cut and "nnz" denotes the number of nonzeros in $A$, i.e., the total number of edges in the adjacency graph; "cut" is the edge cut of interest. 
TABLE 4.1

Relative cut sizes and amounts of the coefficient information discarded to construct preconditioners for problem (4.1) with coefficients in (4.2).

\begin{tabular}{|c||c|c||c|c|}
\cline { 2 - 5 } \multicolumn{1}{c|}{} & \multicolumn{2}{c||}{2 subdomains } & \multicolumn{2}{c|}{ 4 subdomains } \\
\hline Partitioning & relcut & relcoef & relcut & relcoef \\
\hline \hline Acut-RSB & 0.76 & $3 \times 10^{-5}$ & 1.25 & 0.42 \\
\hline MeTiS $(y)$ & 0.44 & 0.85 & 0.86 & 1.62 \\
\hline MeTiS $(t)$ & 1.06 & $4 \times 10^{-5}$ & 1.67 & 0.88 \\
\hline MeTiS(no w. $)$ & 0.48 & 1.07 & 1.01 & 2.13 \\
\hline
\end{tabular}

We see that the cut sizes corresponding to Acut-RSB are relatively large in terms of the edge count, compared to the unweighted MeTiS and $\operatorname{MeTiS}(y)$. At the same time, the amount of the coefficient information discarded for preconditioning is significantly smaller. The similar properties are exhibited by $\operatorname{MeTiS}(t)$. Note that, in the case of bipartitioning, "relcoef" for Acut-RSB is only slightly smaller than that for $\operatorname{MeTiS}(t)$, whereas the quality of the associated preconditioner is much higher; see Figure 4.4 (left).

To the best of our knowledge, there are no available convergence bounds that are based on "relcoef". However, from the practical point of view, it is reasonable to expect that the smaller "relcoef" the higher is the quality of the corresponding preconditioner. In particular, if a standard graph partitioner is at hand, then common approaches for assigning the edge weights, such as in $\operatorname{MeTiS}(t)$, are motivated exactly by this heuristic. While often achieving the smallest values of "relcoef" indeed leads to better results, we demonstrate that the dependence does not hold in general.

More precisely, we keep track of "relcoef" to show that the preconditioning quality is affected not only by the sum of (absolute values of) the discarded coefficients, but also by the actual combination of edges in the cut. While the traditional MeTiS based approaches can succeed in the former, Acut-RSB is capable of choosing more suitable edge combinations for the resulting cuts. This point is further elaborated on in the following examples.

2D diffusion equation: the "checkerboard" jump location. Let us now consider a discretization of equation (4.1) with zero Dirichlet boundary conditions, where the jumps in coefficients are located in the "checkerboard" fashion,

$$
a(x, y)=b(x, y)=\left\{\begin{array}{cl}
10^{5}, & \text { "black" } \\
1, & \text { "white" }
\end{array}\right.
$$

as shown in Figure 4.5. As in the above example, we use a 5-point FD stencil on a 128-by-128 uniform grid, which leads to an SPD linear system of size $n=16,384$.

The results of bipartitioning produced by different methods are presented in Figure 4.6. Similar to the previous example, Acut-RSB perfectly detects the jump regions and avoids "cutting" inside. Note that the resulting two subdomains are disconnected. The eigenvector of (3.18), used to define the Acut-RSB bipartition, is shown in Figure 4.8 (right). The parameters $\gamma$ and $\delta$ are the same as in the previous example, i.e., $10^{5}$ and 1 , respectively. The LOBPCG convergence tolerance is set to $10^{-7}$.

Figure 4.6 demonstrates that, unlike the unweighted MeTiS, the runs of $\operatorname{MeTiS}(y)$ and $\operatorname{MeTiS}(t)$ deliver partitions that adapt to the geometry of the jumps, i.e., attempt to follow the boundaries of the "black" subregions. The advantage, with respect to convergence of PCG-AS, of using matrix coefficients at the partitioning stage is 


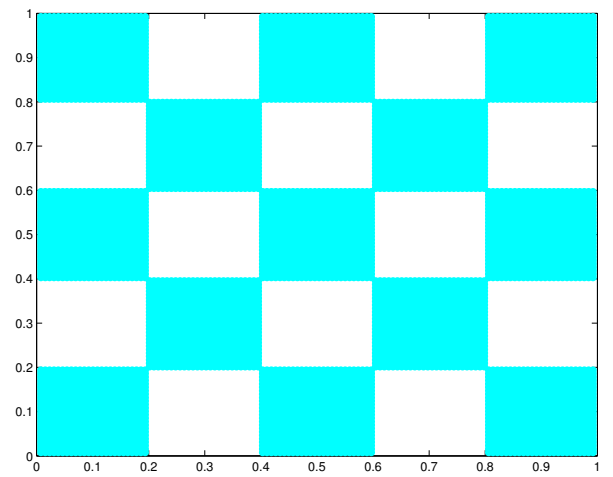

FIG. 4.5. The "checkerboard" location of the jump in coefficients (4.3) for problem (4.1). The shaded (cyan for the color plot) subdomains correspond to the "black" jump regions.
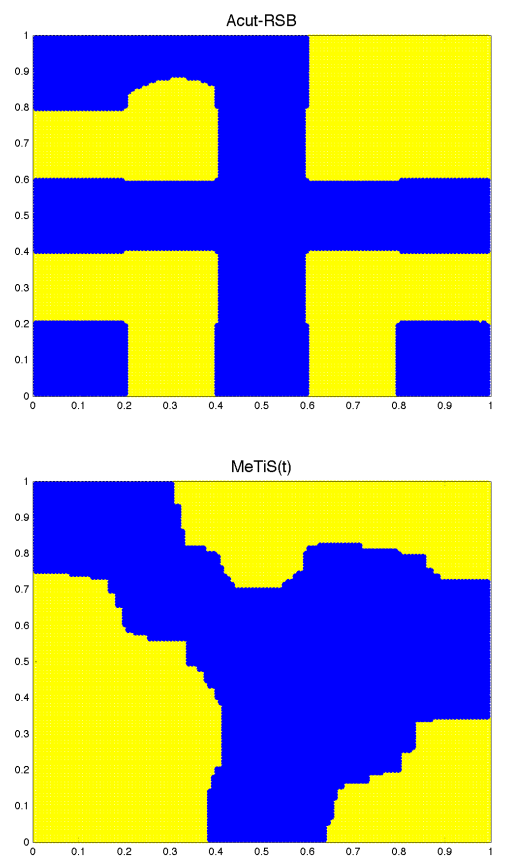
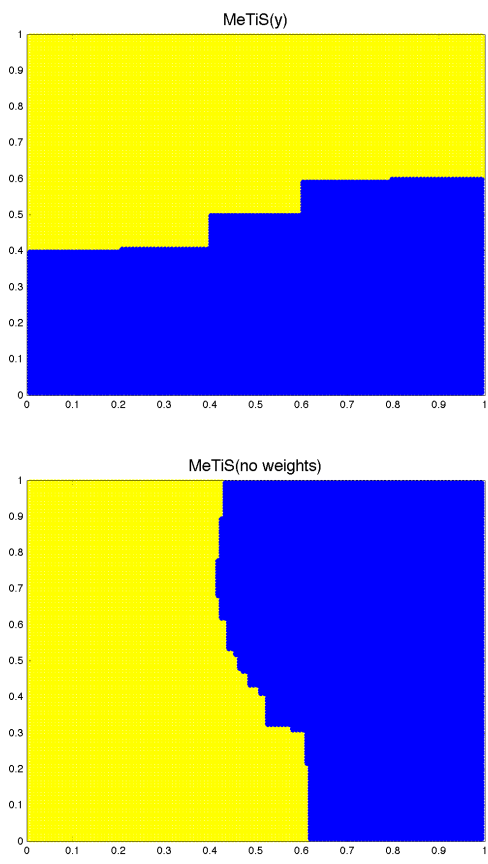

FIG. 4.6. Bipartitions for problem (4.1) with coefficients in (4.3).

further confirmed in Figure 4.7.

Figure 4.7 shows that, in terms of iteration count, PCG-AS with Acut-RSB slightly outperforms the analogues based on $\operatorname{MeTiS}(y)$ and $\operatorname{MeTiS}(t)$. Interestingly, however, the coefficient sum of (absolute values of) the matrix coefficients discarded to construct the preconditioner, reported in Table 4.2, is not the smallest for AcutRSB. More precisely, the "relcoef" value corresponding to Acut-RSB is three times larger than that of $\operatorname{MeTiS}(t)$. This observation clearly allows one to conclude that the preconditioning quality is affected not only by the magnitudes of the discarded matrix entries, but also by the specific combination of edges selected to the cut that defines the underlying partitions. 


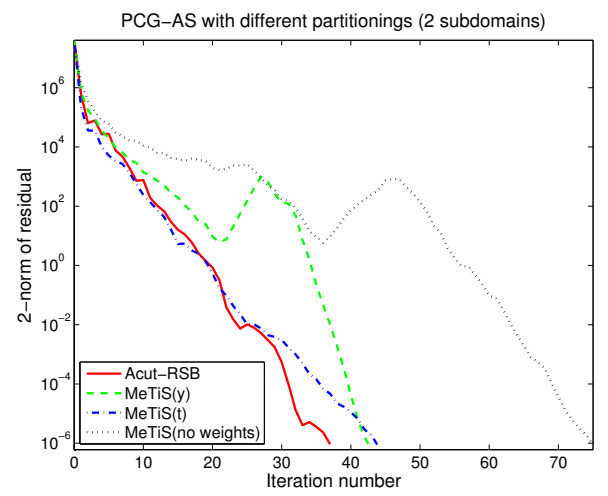

FIG. 4.7. Convergence of $P C G-A S$ with different bipartitions for problem (4.1) with coefficients in (4.3). The linear system is of size $n=16,384$.

TABLE 4.2

Relative cut sizes and amounts of the coefficient information discarded to construct preconditioners for bipartitioning problem (4.1) with coefficients in (4.3).

\begin{tabular}{|l||c|c|}
\hline Partitioning & relcut & relcoef \\
\hline \hline Acut-RSB & 1.78 & 0.06 \\
\hline $\operatorname{MeTiS}(y)$ & 0.47 & 0.17 \\
\hline $\operatorname{MeTiS}(t)$ & 1.23 & 0.02 \\
\hline MeTiS(no w.) & 0.48 & 0.47 \\
\hline
\end{tabular}

3D linear elasticity. Our next experiment is based on the example constructed by Mandel et al. in [25] to test the performance of the adaptive Balancing Domain Decomposition by Constraints (BDDC) [5] in three dimensions. In this example, the authors consider the 3D linear elasticity problem (see, e.g., [18]) in a cube with material parameters $E=10^{6} \mathrm{~Pa}$ and $\nu=0.45$, penetrated by four bars with parameters $E=2.1 \times 10^{11} \mathrm{~Pa}$ and $\nu=0.3$; see Figure 4.9. Zero Dirichlet boundary conditions are assumed.

The problem has been discretized using bilinear finite elements (FE), resulting in 107,811 degrees of freedom. In our tests, we apply different schemes to partition the problem into 8 and 16 subdomains, and assess the quality of the obtained preconditioners according to the number of PCG-AS iterations. The parameters $\gamma$ and $\delta$ for $\operatorname{MeTiS}(y)$ and $\operatorname{MeTiS}(t)$ have been set to $10^{4}$ and $10^{-4}$, respectively. The LOBPCG convergence tolerance is $10^{-4}$. In the case of 16 subdomains, on the bottom level of the Acut-RSB recursion (after producing 8 subdomains), we decrease the tolerance to $10^{-7}$. The right-hand side $b$ is chosen as a random unit vector.

Figure 4.10 shows that Acut-RSB leads to the best convergence of PCG-AS with both 8 and 16 subdomains. Note that, according to the results reported in Table 4.3, the cut size produced by Acut-RSB is about 2-2.5 times larger than that delivered by the MeTiS runs, implying extra communication overhead if implemented in parallel. The gain from this "loss of parallelism", however, is the (approximately) $10 \%$ to $45 \%$ decrease in the iteration count compared to the closest competitor, $\operatorname{MeTiS}(t)$, and more than (approximately) 60\% decrease compared to the unweighted MeTiS. Once again, from Table 4.3, we note that for 16 subdomains "relcoef" for Acut-RSB is larger than that for $\operatorname{MeTiS}(t)$, although the convergence of the former is better. 

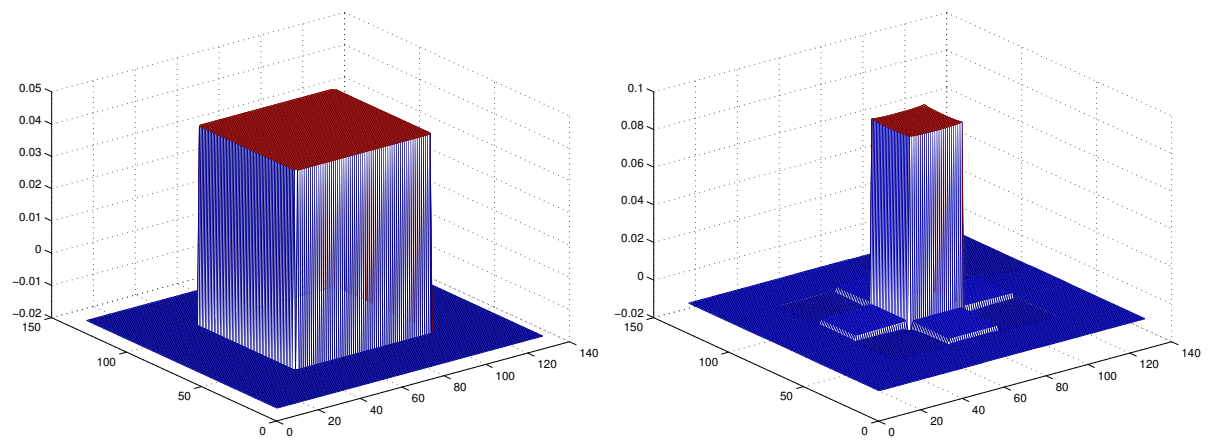

FIG. 4.8. "Mesh" plot of the eigenvector $v$ of (3.18) used for bipartitioning problem (4.1) with coefficients in (4.2) (left) and (4.3) (right). Both eigenvectors capture the discontinuities in coefficients of the corresponding problems.

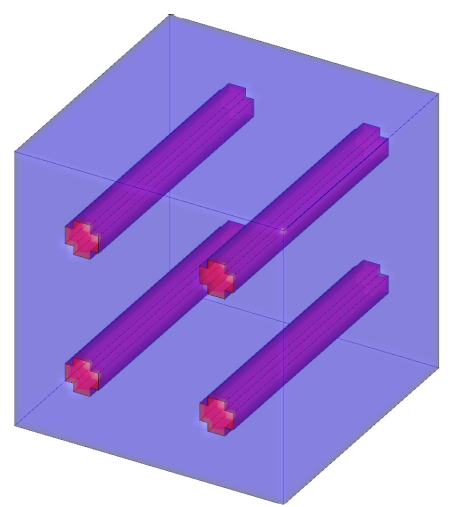

Fig. 4.9. 3D linear elasticity: cube with jumps in coefficients. Example by Mandel et al. [25].

2D diffusion equation: unstructured grid. In this concluding example, we consider a FE discretization of diffusion equation (4.1) on an unstructured grid. We assume Dirichlet boundary conditions and $f(x, y)=1$. The problem domain represents a unit square with four inscribed circles of the same radius; see Figure 4.11 (left).

In order to discretize the equation on an unstructured grid, we use MATLAB PDE Toolbox. The toolbox allows one to define the problem geometry as well as the PDE coefficients and to introduce an initial triangulation, which is further modified by a few refinement steps. The latter results in an unstructured FE mesh with 47, 713 degrees of freedom, shown in Figure 4.11 (right).

The goal of the current example is twofold. On the one hand, we show that AcutRSB can be successfully applied to problems on unstructured grids. On the other hand, the example reveals potential difficulties with the new partitioning strategy, which should be addressed in future research.

Let us first consider the following definition of the coefficients:

$$
a(x, y)=b(x, y)=\left\{\begin{array}{cl}
10^{7}, & \text { "outside circles and on circles' boundaries" } \\
1, & \text { "inside circles" ; }
\end{array}\right.
$$

i.e., $a(x, y)$ and $b(x, y)$ are the piecewise constants taking a large value outside of the four circles and a small value inside. We partition the problem into two and four 

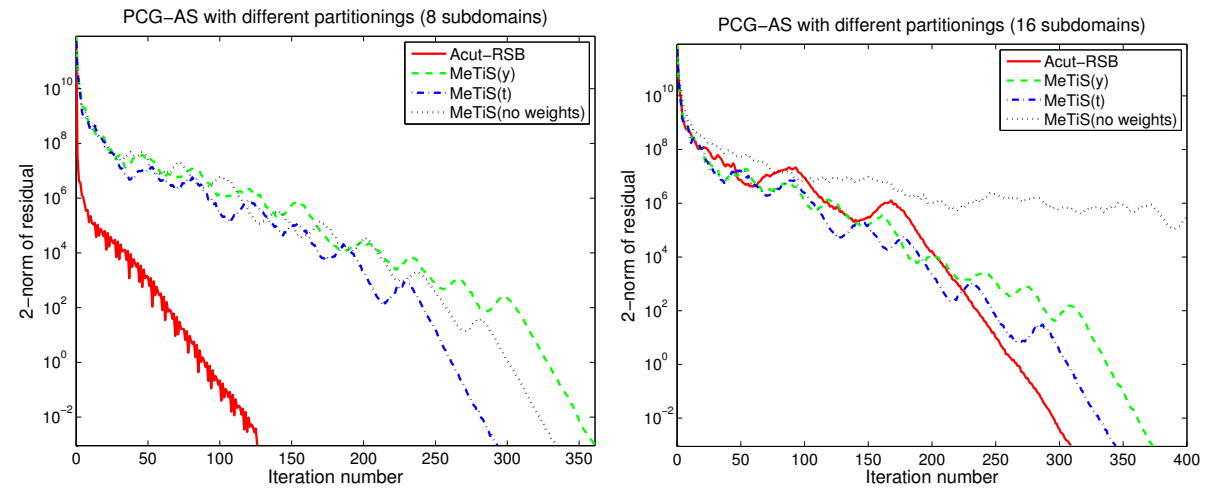

FIG. 4.10. Convergence of $P C G-A S$ with different partitions (8 (left) and 16 (right) subdomains) for the $3 D$ linear elasticity in a cube with large jumps in coefficients; see Figure 4.9. The size of the linear system arising from the FE discretization is $n=107,811$.

TABLE 4.3

Relative cut sizes and amounts of the coefficient information discarded to construct preconditioners for $3 D$ linear elasticity problem in a cube with jumps in coefficients; see Figure 4.9.

\begin{tabular}{|c||c|c||c|c|}
\cline { 2 - 5 } \multicolumn{1}{c||}{} & \multicolumn{2}{c||}{8 subdomains } & \multicolumn{2}{c|}{ 16 subdomains } \\
\hline Partitioning & relcut & relcoef & relcut & relcoef \\
\hline \hline Acut-RSB & 19.98 & 0.01 & 25.39 & 1.55 \\
\hline MeTiS $(y)$ & 8.03 & 2.51 & 11.14 & 2.51 \\
\hline $\operatorname{MeTiS}(t)$ & 7.30 & 1.50 & 11.99 & 1.51 \\
\hline MeTiS(no w.) & 6.77 & 2.24 & 10.61 & 10.28 \\
\hline
\end{tabular}

subdomains using different partitioning schemes and observe the convergence of the corresponding PCG-AS runs. The parameters $\gamma$ and $\delta$ for $\operatorname{MeTiS}(y)$ and $\operatorname{MeTiS}(t)$ are set to $10^{4}$ and $10^{-2}$, respectively. The LOBPCG convergence tolerance is $10^{-7}$ for the case of two, and $10^{-8}$ for the case of four, subdomains. The partitioning results are given in Figures 4.12 and 4.13 .

Figure 4.14 shows that the use of Acut-RSB reduces the iteration count by approximately $15 \%$ compared to the weighted MeTiS runs for the case of two subdomains, and gives a result comparable to $\operatorname{MeTiS}(t)$ for four subdomains. Note that in the latter case the comparable convergence results are produced even though the "relcoef" for $\operatorname{MeTiS}(t)$ is 6 times larger than that of Acut-RSB, as can be seen from Table 4.4.

Now, let us "invert" the definition of the coefficients, so that

$$
a(x, y)=b(x, y)=\left\{\begin{array}{cl}
10^{7}, & \text { "inside circles and on circles' boundaries" } \\
1, & \text { "outside circles" ; }
\end{array}\right.
$$

i.e., $a(x, y)$ and $b(x, y)$ are the piecewise constants taking a small value outside of the four circles and a large value inside. The problem geometry and the mesh are the same as in Figure 4.11. The parameters $\gamma, \delta$, and LOBPCG convergence tolerance remain unchanged. As above, the mesh is partitioned into 2 and 4 subdomains. The results of bipartitioning are shown in Figure 4.15.

Figure 4.16 demonstrates that for the above choice of problem coefficients AcutRSB delivers partitions that fail to produce a satisfactory preconditioning quality. The convergence of the corresponding PCG-AS with Acut-RSB runs is noticeably 

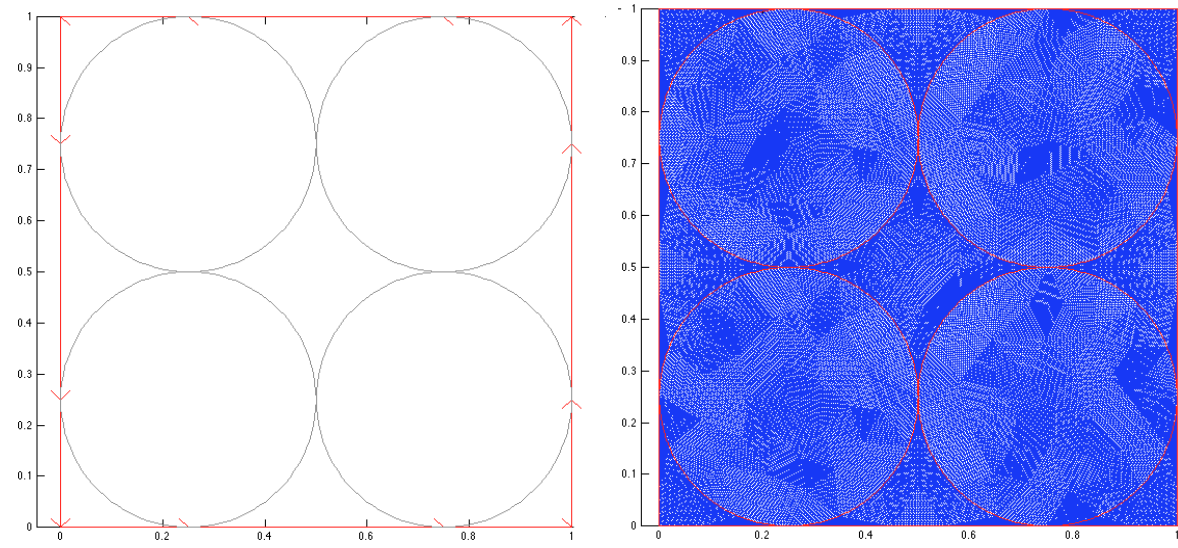

FIG. 4.11. Problem domain (left) and the corresponding FE mesh (right) for equation (4.1).
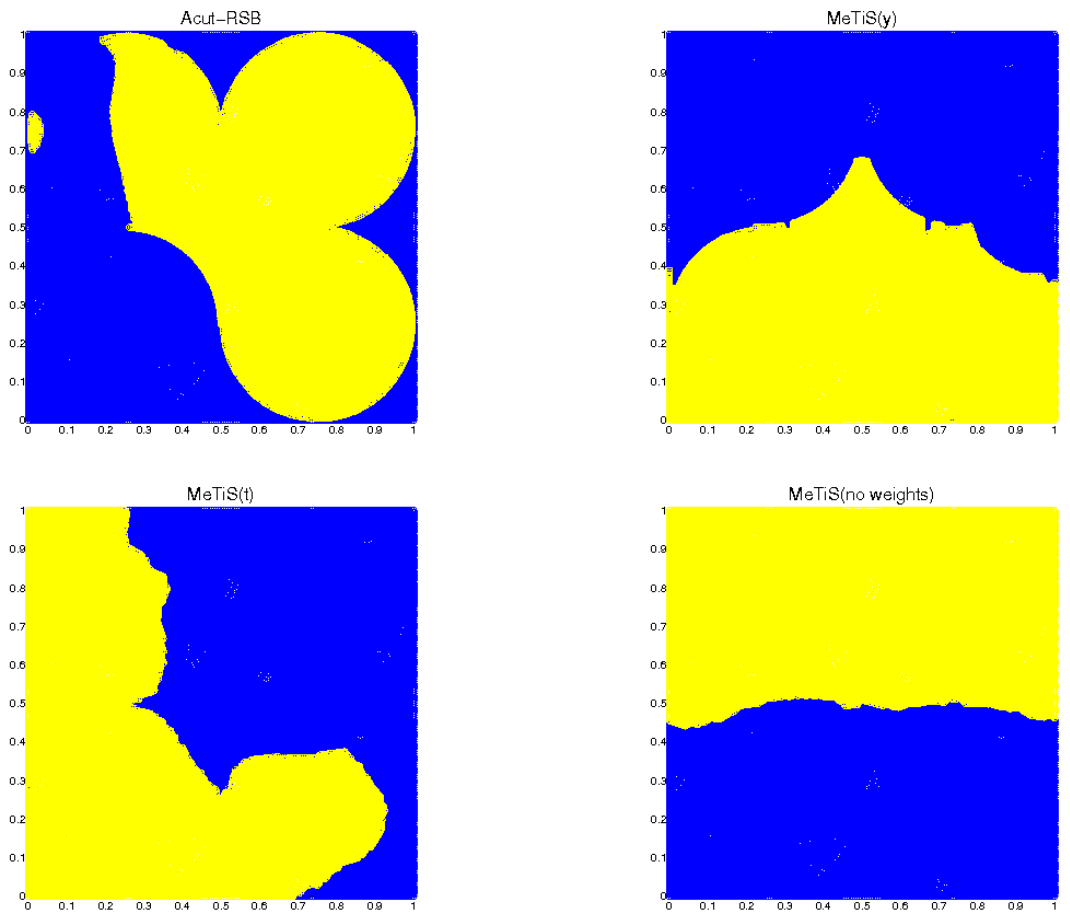

FIG. 4.12. Bipartitions of unstructured mesh (Figure 4.11) for problem (4.1) with coefficients in (4.4).

inferior to that of PCG-AS with MeTiS partitions.

We explain this poor behavior by presence of a large number of edges that connect vertices on the circles' boundaries and those outside the circles; see finer mesh regions along the exterior of the circles' boundaries in Figure 4.11 (right). According to (4.5), such vertices belong to subdomains with different coefficient magnitudes. Therefore, by definition of weights $w_{i j}$, the corresponding edges are the ones targeted 

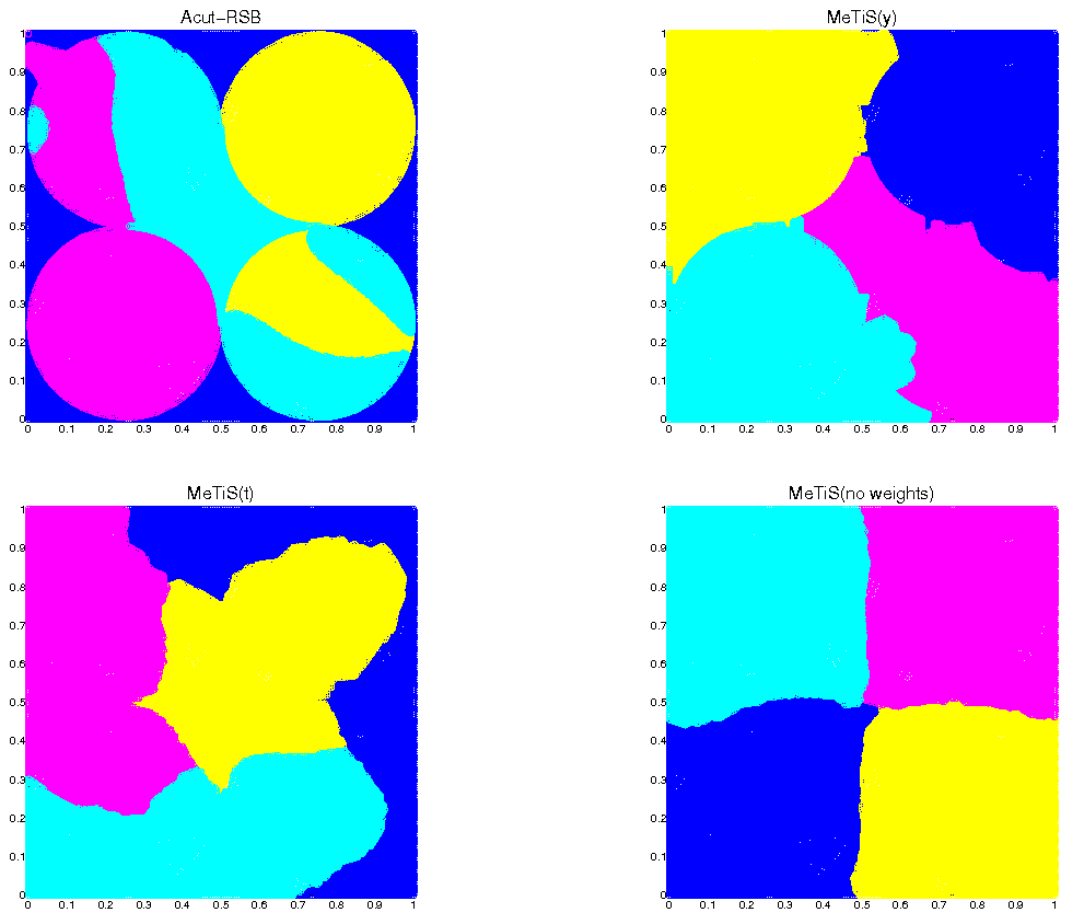

FIG. 4.13. Partitioning of unstructured mesh (Figure 4.11) into 4 subdomains for problem (4.1) with coefficients in (4.4).
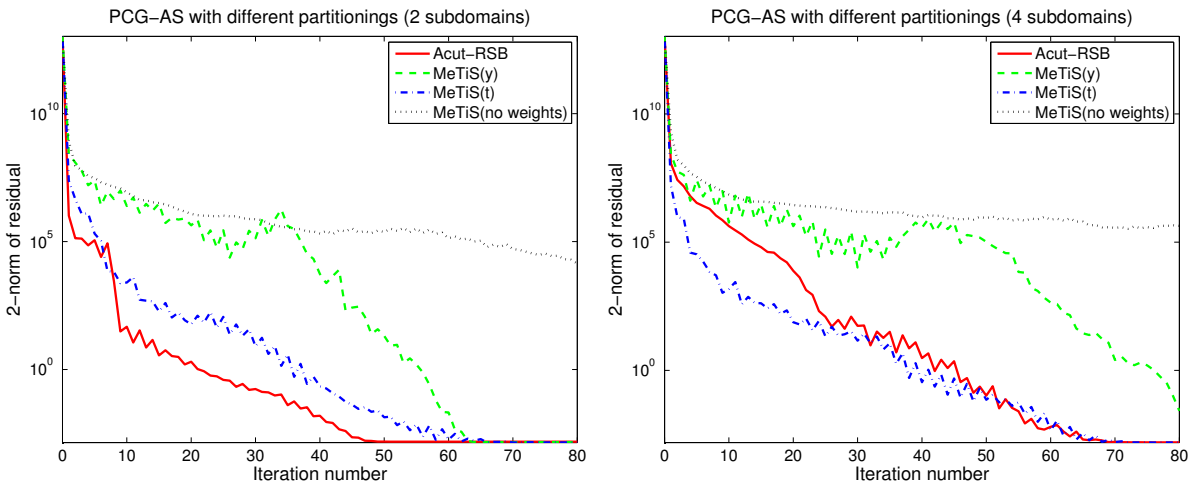

FIG. 4.14. Convergence of $P C G-A S$ with different partitions for problem (4.1) with coefficients in (4.4). The domain and the corresponding FE mesh are given in Figure 4.11. The linear system of size $n=47,713$ is partitioned into 2 (left) and 4 (right) subdomains.

by Acut-RSB; see Figure 4.15 (top left). As a result, the cuts produced by Acut-RSB turn out to be significantly larger (about 5-6 times) than those made by MeTiS; see "relcut" in Table 4.5. Even though the entries $\left|a_{i j}\right|$ corresponding to the edges in Acut are relatively small, they accumulate into an excessively large amount of information discarded from $A$ (see "relcoef" in Table 4.5), thereby leading to the inferior convergence of PCG-AS. In other words, in this example, minimization of (3.10) is mainly 
TABLE 4.4

Relative cut sizes and amounts of the coefficient information discarded to construct preconditioners for problem (4.1) with coefficients in (4.4); see Figure 4.11.

\begin{tabular}{|c||c|c||c|c|}
\cline { 2 - 5 } \multicolumn{1}{c|}{} & \multicolumn{2}{c||}{2 subdomains } & \multicolumn{2}{c|}{ 4 subdomains } \\
\hline Partitioning & relcut & relcoef & relcut & relcoef \\
\hline \hline Acut-RSB & 1.03 & $3 \times 10^{-4}$ & 1.96 & 0.06 \\
\hline MeTiS $(y)$ & 0.46 & 0.10 & 0.85 & 0.16 \\
\hline MeTiS $(t)$ & 0.53 & $5 \times 10^{-4}$ & 1.04 & 0.01 \\
\hline MeTiS(no w. $)$ & 0.28 & 0.47 & 0.56 & 1.24 \\
\hline
\end{tabular}
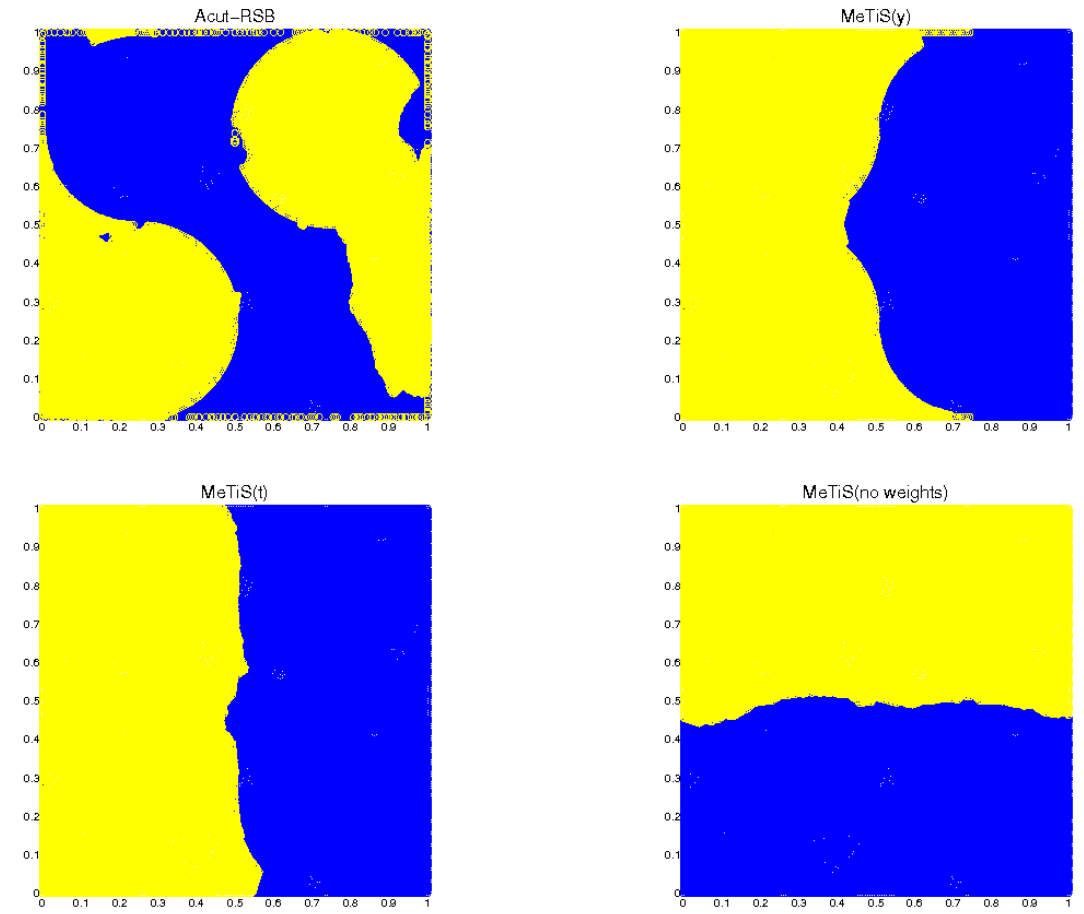

FIG. 4.15. Bipartitions of unstructured mesh (Figure 4.11) for problem (4.1) with coefficients in $(4.5)$.

contributed by the increase of the number of cut edges rather than the decrease of their weight, which appears to hinder the convergence.

In contrast, the number of edges that connect vertices on the circles' boundaries and those inside the circles is relatively small; see coarser mesh regions along the interior of the circles' boundaries in Figure 4.11 (right). If problem coefficients are defined by (4.4), then these are the edges targeted by Acut-RSB; see Figure 4.12 (top left). However, their number is smaller than that of the edges discraded by the (unsuccessful) run of Acut-RSB with coefficients (4.5). Therefore, as reported in Table 4.4, the values of "relcut" and "relcoef" corresponding to Acut-RSB are not large, i.e., minimization of (3.10) is given by a suitable balance between the cut weight and the number of cut edges. As a result, a better convergence behavior is observed in Figure 4.14. 

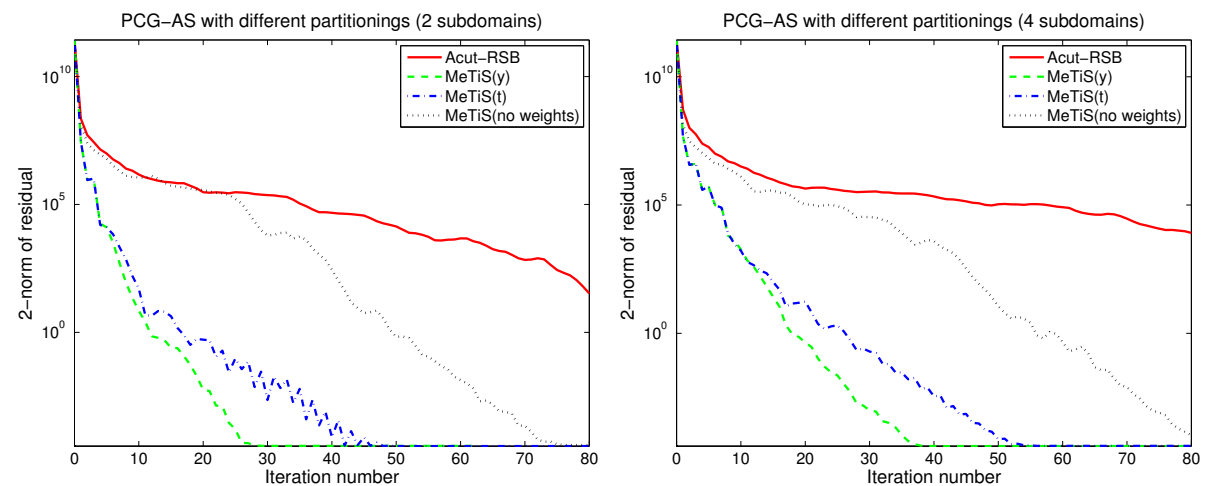

FIG. 4.16. Convergence of $P C G-A S$ with different partitions for problem (4.1) with coefficients in (4.5). The domain and the corresponding FE mesh are given in Figure 4.11. The linear system of size $n=47,713$ is partitioned into 2 (left) and 4 (right) subdomains.

TABLE 4.5

Relative cut sizes and amounts of the coefficient information discarded to construct preconditioners for problem (4.1) with coefficients in (4.5); see Figure 4.11.

\begin{tabular}{|c||c|c||c|c|}
\cline { 2 - 5 } \multicolumn{1}{c|}{} & \multicolumn{2}{c||}{ 2 subdomains } & \multicolumn{2}{c|}{ 4 subdomains } \\
\hline Partitioning & relcut & relcoef & relcut & relcoef \\
\hline \hline Acut-RSB & 1.80 & 0.44 & 3.67 & 1.51 \\
\hline $\operatorname{MeTiS}(y)$ & 0.38 & 0.01 & 0.76 & 0.01 \\
\hline $\operatorname{MeTiS}(t)$ & 0.27 & 0.01 & 0.54 & 0.01 \\
\hline MeTiS(no w.) & 0.28 & 0.10 & 0.56 & 0.11 \\
\hline
\end{tabular}

Finally, let us remark that, unlike in all the previous examples, the best convergence in Figure 4.16 is given by PCG-AS with MeTiS $(y)$. In particular, this shows that it is not clear how to optimally define MeTiS weights if the preconditioning quality becomes an objective. We also note that the values of "relcoef" in Table 4.5 for $\operatorname{MeTiS}(y)$ and $\operatorname{MeTiS}(t)$ are essentially the same (up to the sixth decimal digit), while the convergence of the former is noticeably superior.

5. Conclusion. This paper introduces a new approach for partitioning SPD linear systems. The suggested technique is based on approximating the so-called Acut of the matrix adjacency graph. The information about matrix coefficients is utilized through the graph's edge weights.

The resulting partitioning procedure represents a form of RSB, where each step of the recursion requires solving a generalized eigenvalue problem that simultaneously involves weighted and standard graph Laplacians. It is shown that, for a number of test problems, the new partitioning significantly improves the quality of the associated nonoverlapping AS preconditioners, compared to MeTiS with several different weighting schemes. In the context of parallel solution, the observed increase in the robustness of the iterative method occurs at a price of extra communication overhead.

The new partitioning strategy is shown to be effective for test linear systems with large variations in matrix coefficients. We have observed that the quality of the result strongly depends on the difference in the magnitude of coefficients, as well as (in terms of PDE's) on the problem geometry and mesh structure. Future research should address the development of practical recommendations on when the proposed 
partitioning method is preferable to the existing techniques. Since at the current exploratory stage the test problems have been relatively small and simple, it is of interest to benchmark the partitoning quality and performance of the new approach at a larger scale and for more complex geometries.

While in this work we have disregarded the requirement on minimizing communication volume, future research should also address the trade-off between preconditioning quality and parallel efficiency. In particular, we hope that this will lead to the containment of the large cut sizes produced by Acut.

This paper considers only the case of nonoverlapping AS (block Jacobi) preconditioners. However, it is of interest to apply the same partitioning approach for other preconditioning strategies. For example, our experiments (not reported here) suggest that results for the overlapping AS are similar to the above reported nonoverlapping AS. The overlaps have been introduced by growing a few layers of nodes for each nonoverlapping subdomain.

The new partitioning procedure is heavily rooted in the SPD properties of the coefficient matrix. Therefore, it is not clear if successful results can be obtained for symmetric indefinite or only structurally symmetric matrices.

Finally, we hope that applications of the Acut based partitioning can be found in other areas of science and engineering, such as data clustering and network analysis and logistics.

Acknowledgments. The authors thank Dr. Bedřich Sousedík for sharing the $\mathrm{FE}$ assembling codes to reproduce the 3D linear elasticity example from [25], and Ruipeng Li for assisting with SPARSKIT matrix generation.

\section{REFERENCES}

[1] O. Axelsson, Iterative Solution Methods, Cambridge University Press, New York, NY, 1994.

[2] U. V. ÇATALyÜrek and C. AYKanat, Hypergraph-partitioning-based decomposition for parallel sparse-matrix vector multiplication, IEEE Trans. Parallel Distrib. Syst., 10 (1999), pp. 673693.

[3] T. H. Cormen, C. E. Leiserson, R. Rivest and C. Stein, Introduction to Algorithms (2nd ed.), MIT Press and McGraw-Hill, 2001.

[4] C. H. Q. Ding, X. He, H. Zha, M. Gu And H. D. Simon, A Min-max cut algorithm for graph partitioning and data clustering, in Proc. 2001 IEEE International Conference on Data Mining (ICDM), 107-114. IEEE Computer Society, Washington (2001).

[5] C. R. Dohrmann, A preconditioner for substructuring based on constrained energy minimization, SIAM J. Sci. Comput., 25 (2003), pp. 246-258.

[6] W. Donath And A. Hoffman, Algorithms for partitioning of graphs and computer logic based on eigenvectors of connection matrices, IBM Technical Disclosure Bulletin, 15 (1972), pp. 938-944.

[7] W. Donath And A. Hoffman, Lower bounds for the partitioning of graphs, IBM J. Res. Develop., 17 (1973), pp. 420-425.

[8] C. M. Fiduccia and R. M. Mattheyses, A linear time heuristic for improving network partitions, in Proc. 19th IEEE Design Automation Conference, 1982, pp. 175-181.

[9] M. Fiedler, Algebraic connectivity of graphs, Czech. Math. J., 23 (1973), pp. 298-305.

[10] M. FIEDLER, A property of eigenvectors of non-negative symmetric matrices and its application to graph theory, Czech. Math. J., 25 (1975), pp. 619-633.

[11] D. Fritzsche, A. Frommer, And D. B. Szyld, Extensions of certain graph-based algorithms for preconditioning, SIAM J. Sci. Comput., 29 (2007), 2144-2161.

[12] T. GoenRIng AND Y. SAAD, Heuristic algorithms for automatic graph partitioning, Tech. rep., Department of Computer Science, University of Minnesota, Minneapolis, 1994.

[13] P. Have, R. Masson, F. Nataf, M. Szydlarski, and T. Zhao, Algebraic domain decomposition methods for highly heterogeneous problems, Tech. rep., INRIA, hal-00611997, ver. 1, 2011. 
[14] B. Hendrickson And T. G. Kolda, Graph partitioning models for parallel computing, Parallel Computing, 26 (2000), pp. 519-1534.

[15] B. Hendrickson and R. Leland, The Chaco User's Guide: Version 2.0, 1994.

[16] B. HENDRICKSON AND R. Leland, An improved spectral graph partitioning algorithm for mapping parallel computations, SIAM J. Sci. Comput., 16 (1995), 452-469.

[17] B. HENDRICKSON AND R. Leland, A multilevel algorithm for partitioning graphs, in Proc. 1995 ACM/IEEE conference on Supercomputing (CDROM), 1995.

[18] T. J. R. Hughes, The Finite Element Method: Linear Static and Dynamic Finite Element Analysis, Dover, 2000.

[19] G. Karypis and V. Kumar, MeTis: Unstructured Graph Partitioning and Sparse Matrix Ordering System, Version 4.0, University of Minnesota, Minneapolis, MN, 2009.

[20] G. KARYPIS AND V. KUMAR, A fast and high quality multilevel scheme for partitioning irregular graphs, SIAM J. Sci. Comput., 20 (1998), 359-392.

[21] G. KARYPIS AND V. Kumar, Multilevel k-way partitioning scheme for irregular graphs, J. Parallel Distr. Com., 48 (1998), 96-129.

[22] B. W. Kernighan and S. Lin, An efficient heuristic procedure for partitioning graphs, Bell Sys. Tech. J., 49 (1970), pp. 291-307.

[23] A. V. KNYAZEv, Toward the optimal preconditioned eigensolver: locally optimal block preconditioned conjugate gradient method, SIAM J. Sci. Comput., 23 (2001), pp. 517-541.

[24] U. LuxBurg, A tutorial on spectral clustering, Stat. Comput., 17 (2007), pp. 395-416.

[25] J. Mandel, B. Sousedík, And J. Šístek, Adaptive BDDC in Three Dimensions, Math. Comput. Simulation, 82 (2012), pp. 1812-1831.

[26] G. L. Miller, S. H. Teng, W. Thurston, and S. A. Vavasis, Automatic mesh partitioning, in Sparse Matrix Computations: Graph Theory Issues and Algorithms, A. George, J. Gilbert, and J. Liu, eds., 1993. IMA Volumes in Mathematics and Its Applications.

[27] B. N. PARlett, The Symmetric Eigenvalue Problem, SIAM, 1998.

[28] F. Pellegrini, SCOTCH 5.1 User's guide, Tech. rep., LaBRI, September 2008.

[29] A. Pothen, H. D. Simon, And K.-P. Liou, Partitioning sparse matrices with eigenvectors of graphs, SIAM J. Matrix Anal. Appl., 11 (1990), pp. 430-452.

[30] Y. SAAD, Iterative Methods for Sparse Linear Systems, SIAM, 2003.

[31] Y. SAAD, SPARSKIT: A basic tool kit for sparse matrix computations, http://www-users.cs. umn.edu/ saad/software/SPARSKIT/index.html, 1994.

[32] Y. SAAD AND M. Sosonkina, Non-standard parallel solution strategies for distributed sparse linear systems, in Parallel Computation: Proc. of ACPC'99, A. U. P. Zinterhof, M. Vajtersic, ed., Lecture Notes in Computer Science, Berlin, 1999, Springer-Verlag.

[33] J. Shi and J. Malik, Normalized cuts and image segmentation, IEEE Trans. Pattern Anal. Mach. Intell., 22 (2000), pp. 888-905.

[34] H. Simon, Partitioning of unstructured problems for parallel processing, Comput. Syst. Eng., 2 (1991), 135-148.

[35] H. Simon And S. H. Teng, How Good is Recursive Bisection?, SIAM Journal on Scientific Computing, 18 (5), pp. 1436-1445 (1997).

[36] B. Smith, P. E. Buørstad and W. Gropp, Domain Decomposition: Parallel Multilevel Methods for Elliptic Partial Differential Equations, Cambridge University Press, 1996.

[37] D. Spielman and S. Teng, Spectral partitioning works: planar graphs and finite element meshes, in 37th Annual Symposium on Foundations of Computer Science (Burlington, VT, 1996), pp. 96-105. CA: IEEE Comput. Soc. Press, Los Alamitos (1996)

[38] C. Walshaw and M. Cross, JOSTLE: Parallel Multilevel Graph-Partitioning Software - An Overview, in Mesh Partitioning Techniques and Domain Decomposition Techniques, ed. F. Magoules, Civil-Comp Ltd., pp. 27-58, 2007.

[39] A. Toselli and O. Widlund, Domain Decomposition Methods - Algorithms and Theory, Springer, 2005 